RESEARCH PAPER RP1252

Part of Journal of Research of the National Bureau of Standards, Volume 23, November 1939

\title{
A PORTABLE APPARATUS FOR DETERMINING THE RELATIVE WEAR RESISTANCE OF CONCRETE FLOORS
}

\author{
By Louis Schuman and John Tucker, Jr.
}

\section{ABSTRACT}

A portable machine for producing rapid wear and an optical gage for measuring the depths of wear were designed and built. Studies were made on 138 slabs of concrete to determine the effect of various mixes and $C / W$ ratios and of such factors as aggregate types and grading, finishing procedures, dust coats, and liquid surface treatments. The tests show that metallic hardeners, dust coats containing cement, and delayed troweling are effective in increasing wear resistance, The test methods used show advantages in the use of dry mixes, coarse aggregates, and of adequate damp curing. The use of a high-early-strength cement permitted the damp curing period to be greatly reduced without decreasing the wear resistance.

\section{CONTENTS}

I. Introduction

II. Apparatus

1. Machine for producing rapid wear

2. Device for measuring depth of wear

III. Outline of investigation

IV. Materials, test specimens, and test methods

1. Materials .

2. Preparation and treatment of test specimens $\ldots \ldots 54$

3. Test methods . .

V. Test results _.

1. General discussion

2. Effect of various factors on wear

(a) Water content and grading of aggregate

(b) Type of aggregate.

(c) Finishing procedure and dust coats .

(d) Cements and curing conditions

(e) Liquid surface treatments _.

3. Pitting .

VI. Summary and conclusions

VII. References

\section{INTRODUCTION}

Wear tests of concretes have been made by many investigators [1 to 7]. ${ }^{1}$ Abrams [2], using the Talbot-Jones rattler, determined the effect of such factors as the water content and the fineness modulus of aggregates on the wear to depths of 2 in. In tests made by the Bureau of Public Roads [4], rubber-tired wheels with chains were used for abrading the surfaces under test; these studies were concerned mainly with the wear resistance of various aggregates. The effect of methods

\footnotetext{
1 Figures in brackets indicate the literature references at the end of this paper.
} 
of finishing and of other factors upon the wear resistance of the surface "skin," that is, to a depth of approximately 0.1 in., were not studied.

The purpose of the present investigation was to develop an apparatus for producing rapid wear and a method for measuring depths of wear up to $0.1 \mathrm{in}$.

\section{APPARATUS}

\section{MACHINE FOR PRODUCING RAPID WEAR}

The machine which was developed is shown in figure 1. It consists essentially of three vertical shafts to which the 3-in.-diameter coldrolled steel abrading disks $A, B$, and $C$ are attached by means of universal joints. Plate $P$, carrying these abrading elements, rotates at $2 \mathrm{rpm}$, while the abrading disks rotate at $180 \mathrm{rpm}$. Cups $L$ attached to the disk shafts were loaded with lead to give a total load on each disk of $25 \mathrm{lb}$. The net abrading area (face, less slots) of each disk was 5.6 in $^{2}$; the pressure was therefore $4.4 \mathrm{lb} / \mathrm{in}^{2}$.

The revolving disks produced measurable wear; but for more rapid wear an abrasive, No. 60 silicon carbide grain, was used. This was fed from cup $D$, through a tube with $1 / 16$-in. diameter opening, and deposited on the surface to be abraded, about $1 / 4$ in. within the outer edge of the track formed by the abrading disks. The rotation of plate $P$ and of the disks causes the abrasive to be swept gradually toward the center and, together with particles loosened from the test slab, to pile up near the plate support. The circular track formed by the simultaneous rotation of the plate and disks has an inside diameter of 12 in. and an outside diameter of 18 in. Preliminary tests showed that, with the loads and abrasive used, depths of wear up to about 0.1 in. could be obtained when the machine was operated for 20 minutes. By measuring the depth of wear after several shorter periods of operation of the machine, it was possible to study the effects of methods of finishing, dust coats, and "hardeners."

\section{DEVICE FOR MEASURING DEPTH OF WEAR}

The device used for measuring the average depth of the abraded surface is shown diagrammatically in figure 2 . The light from a straight-filament 35-watt galvanometer lamp, $A$, is reflected by a flat chromium-plated reflector, $B$. Though reflection takes place over the whole area of the reflector, the light beam which just passes the straight edge, $E$, placed across the abraded surface affords a measure of the depth of wear. The beams above this "measuring beam" will not appear beyond the straight edge; the beams below it illuminate the worn surface up to a contour, $C$, which is a magnified profile of a radial section of the worn surface. The distance, $m$, from straight edge $E$ to any point, $P$, on this contour $C$ is read on a scale, $S$, ruled on a celluloid plate and graduated in $0.1 \mathrm{in}$. The magnification is the distance, $d$, divided by the height, $h$, above the original surface. Thus the depth of wear at a point, $P$, equals $m \div d / h$. The average distance of contour $C$ from straight edge $E$ was estimated by inspection and used to calculate the depth of wear. The distances $h$ and $d$ were measured directly; in the device used the magnification $d / h$ was 32 , and one division on the scale corresponded to a depth of wear of $0.1 / 32$, or 0.003 in. 

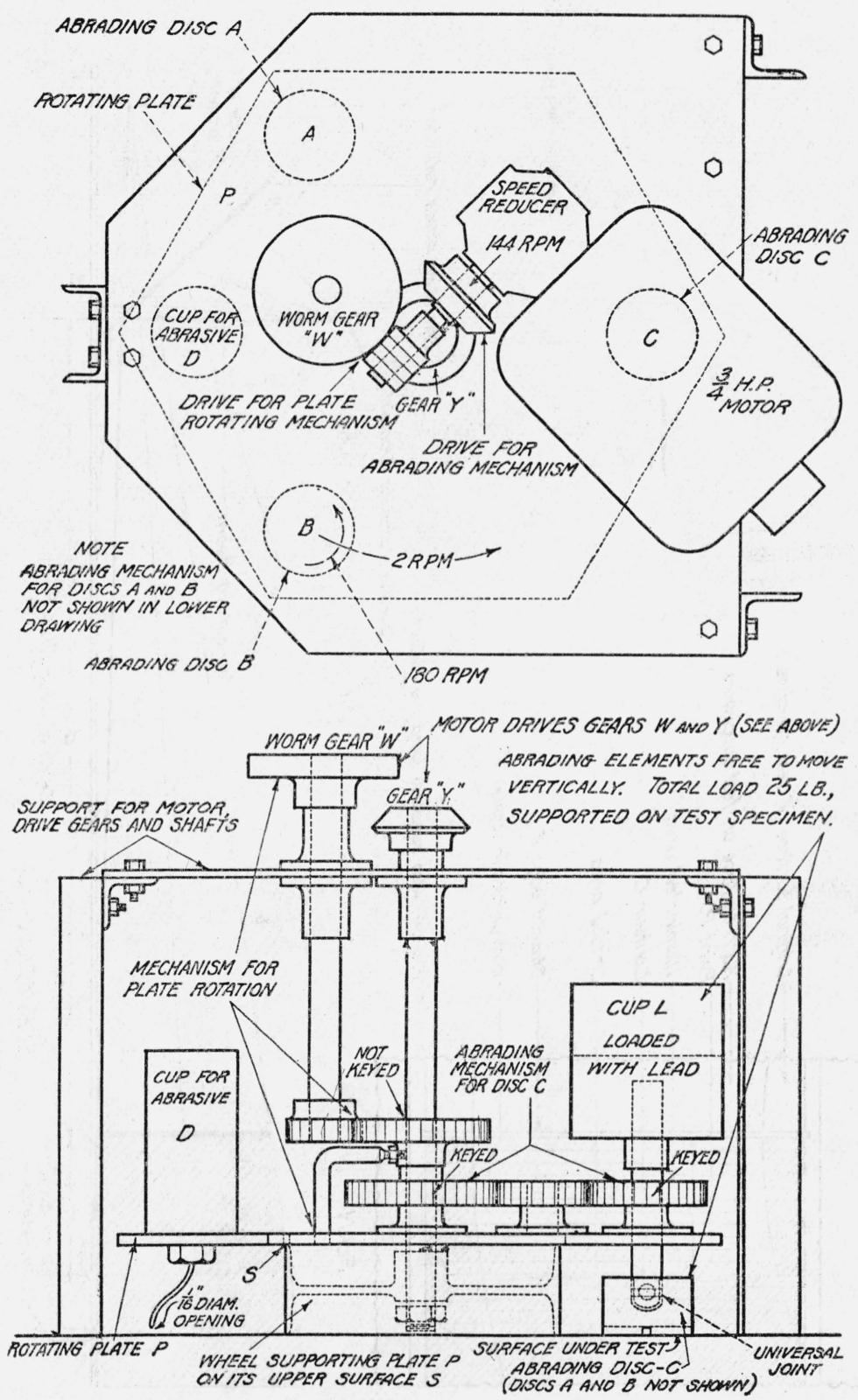

Figure 1.-Abrasion apparatus. 


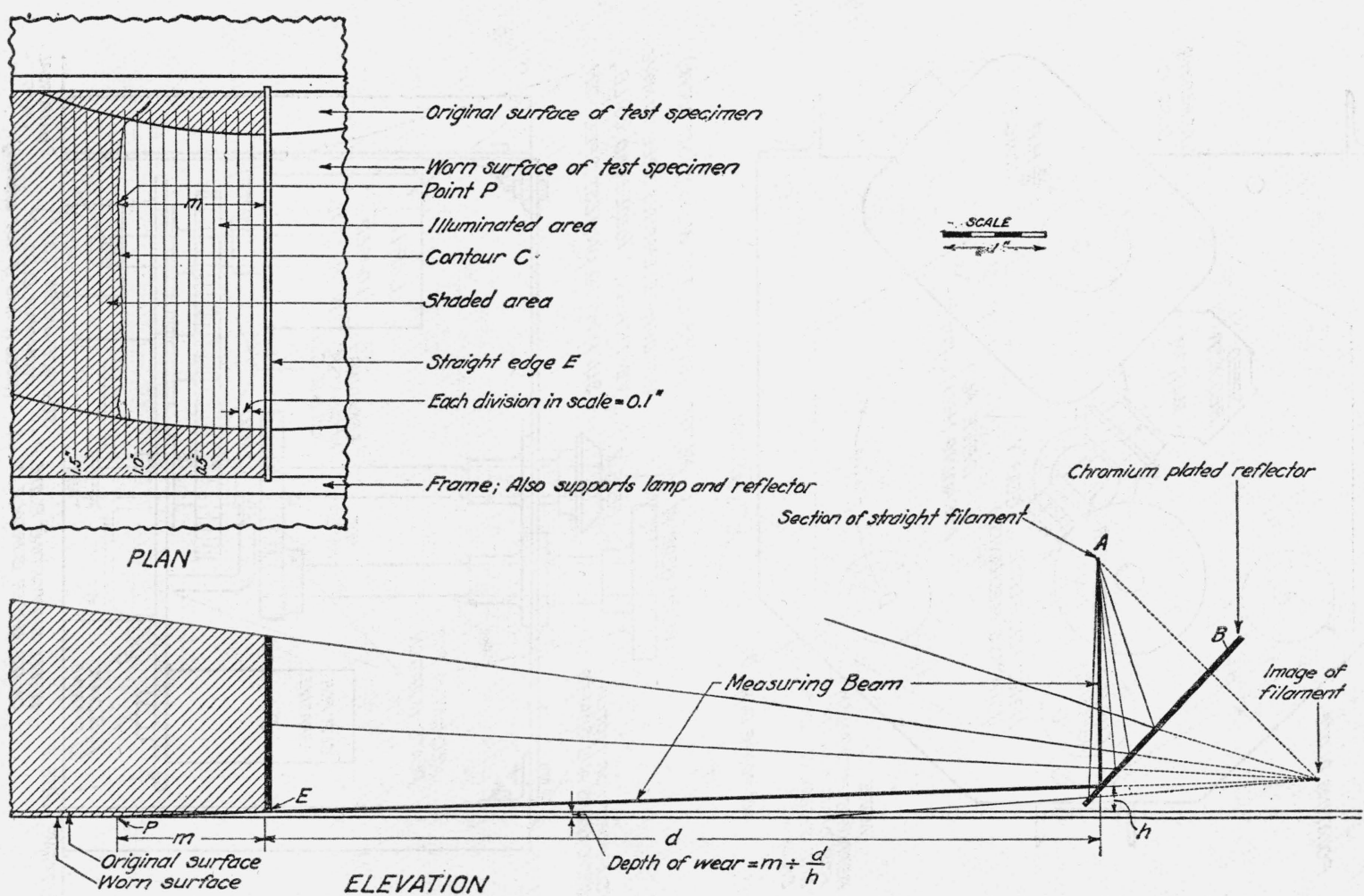

$\rightarrow$

(2)

है

\$.

\& 


\section{OUTLINE OF INVESTIGATION}

The reliability of the apparatus for determining the relative resistance to wear of various surfaces was studied by means of tests on a series of concrete slabs of mixes varying from a $1: 2$ mortar to a $1: 3: 6$ concrete, in which various cements, $C / W$ ratios, sands, and coarse aggregates were used. The surfaces were finished by different methods, such as the application of dust coats of cement, cement and sand, or cement and metallic aggregate. The effects of various time intervals between placing and troweling, and of variation in periods of damp curing were also studied. The effects of sodium silicate and magnesium fluosilicate solutions, two types of materials frequently used for the treatment of concrete surfaces, were studied.

\section{MATERIALS, TEST SPECIMENS, AND TEST METHODS}

\section{MATERIALS}

A "normal" portland cement meeting the requirements of Federal Specification SS-C-191a was used in most of the specimens. This cement contained 2.5 percent of $\mathrm{Fe}_{2} \mathrm{O}_{3}$ and 6.9 percent of $\mathrm{Al}_{2} \mathrm{O}_{3}$. Three slabs were made with a cement also meeting the requirements of the specification but with an $\mathrm{Fe}_{2} \mathrm{O}_{3}$ content of 4.6 percent and an $\mathrm{Al}_{2} \mathrm{O}_{3}$ content of 4.2 percent, and eight slabs were made with a highearly-strength cement meeting the requirements of Federal Specification SS-C-201.

The gradings of the various aggregates used are shown in table 1. Sands $A, B, C, D$, and $E$ and the gravels, all from the Potomac River, were largely silicious. The "traprock" sand $(F)$ and the No. 8 to 3/2-in. "traprock" were from a Pennsylvania quarry. The No. 8 to 8/ -in. crushed limestone was from Martinsburg, W. Va.

TABLE 1.-Grading of aggregates

[Percentage retained on various sieves]

\begin{tabular}{|c|c|c|c|c|c|c|c|c|c|c|c|c|}
\hline \multirow{3}{*}{ Sieve } & \multicolumn{7}{|c|}{ Fine aggregate } & \multicolumn{5}{|c|}{ Coarse aggregate } \\
\hline & \multicolumn{5}{|c|}{ Potomac River sand } & \multirow{2}{*}{$\begin{array}{c}\text { "Trap- } \\
\text { rock" } \\
\text { sand, } \\
F\end{array}$} & \multirow{2}{*}{$\begin{array}{l}\text { Metal- } \\
\text { lic } \\
\text { aggre- } \\
\text { gate }^{b}\end{array}$} & \multicolumn{3}{|c|}{ Potomac River gravel } & \multirow{2}{*}{$\begin{array}{l}\text { Crushed } \\
\text { lime- } \\
\text { stone, } \\
\text { No. } 8 \\
\text { to } 36 \text { in. }\end{array}$} & \multirow{2}{*}{$\begin{array}{l}\text { Crushed } \\
\text { "trap" } \\
\text { rock", } \\
\text { No. } 8 \\
\text { to } 1 / 2 \text { in. }\end{array}$} \\
\hline & $A$ & $B$ & $C$ & $D^{a}$ & $E^{a}$ & & & $\begin{array}{l}\text { No. } 8 \\
\text { to } \\
1 / 4 \text { in. }\end{array}$ & $\begin{array}{l}1 / 4 \text { in. } \\
\text { to } \\
3 / 8 \text { in. }\end{array}$ & $\begin{array}{l}1 / 4 \text { in. } \\
\text { to } \\
3 / 4 \text { in. }\end{array}$ & & \\
\hline $\begin{array}{l}8 / 4 \text { in } \\
88 \text { in } \\
\text { No. } 4 \\
\text { No. } 8 \\
\text { Nc. } 16 \\
\text { No. } 30 \\
\text { No } 50 \\
\text { No. } 100\end{array}$ & $\begin{array}{r}\% \\
0 \\
0 \\
1 \\
9 \\
16 \\
37 \\
86 \\
98\end{array}$ & $\begin{array}{r}\% \\
0 \\
0 \\
1 \\
7 \\
20 \\
28 \\
80 \\
98\end{array}$ & $\begin{array}{l}\% \\
0 \\
0 \\
4 \\
27 \\
43 \\
57 \\
79 \\
98\end{array}$ & $\begin{array}{r}\% \\
0 \\
0 \\
6 \\
56 \\
100 \\
100 \\
100 \\
100\end{array}$ & $\begin{array}{r}\% \\
0 \\
0 \\
0 \\
0 \\
0 \\
0 \\
78 \\
97\end{array}$ & $\begin{array}{r}\% 0 \\
0 \\
1 \\
16 \\
35 \\
50 \\
66 \\
81\end{array}$ & $\begin{array}{r}\% \\
0 \\
0 \\
0 \\
0 \\
6 \\
49 \\
94 \\
99\end{array}$ & $\begin{array}{r}\% 0 \\
0 \\
0 \\
61 \\
94 \\
99 \\
100 \\
100 \\
100\end{array}$ & $\begin{array}{r}\% \\
0 \\
0 \\
100 \\
100 \\
100 \\
100 \\
100 \\
100\end{array}$ & $\begin{array}{r}\% 0 \\
50 \\
100 \\
100 \\
100 \\
100 \\
100 \\
100\end{array}$ & $\begin{array}{r}\% \\
0 \\
0 \\
66 \\
99 \\
99 \\
99 \\
100 \\
100\end{array}$ & $\begin{array}{r}\% \\
0 \\
16 \\
72 \\
98 \\
100 \\
100 \\
100 \\
100\end{array}$ \\
\hline Fineness modulus. . & 2.47 & 2. 44 & 3.08 & 4. 62 & 1. 75 & 2.49 & 2.48 & 5.54 & 6.00 & 6.50 & 5.63 & 5.86 \\
\hline
\end{tabular}

a Prepared from sand $A$ by screening.

b Metallic aggregate is to be distinguished from so-called metallic waterproofing compounds, the former being coarser and free of salts, as well as of oil, gr ease, and nonferrous metals. 
In the study of liquid surface treatments, three grades of sodium silicate were used: The first is designated water glass and was not analyzed. The second had a soda-silica ratio of $1: 3.13$ and is designated high-silica water glass. The third had a soda-silica ratio of 1:1.6 and is designated low-silica water glass. Each of the three grades was diluted to an 11-percent solution by weight. The magnesium fluosilicate "hardener" solution was obtained in two strengths - a 15-percent solution (based on the hydrated salt $\mathrm{MgSiF}_{6} \cdot 6 \mathrm{H}_{2} \mathrm{O}$ ) which was applied without diluting, and a 22-percent solution which was diluted to various concentrations before application

\section{PREPARATION AND TREATMENT OF TEST SPECIMENS}

The test specimens were slabs 23.5 by 27.5 in., usually 1 in. thick; when the aggregate coarser than 12 in. was used, slabs 2 in. thick were made. The materials were stored and the specimens made in a laboratory maintained at $70^{\circ} \pm 2^{\circ} \mathrm{F}$. The materials were mixed in either a laboratory batch mixer or a small concrete drum mixer. Mixing was in all cases continued for 3 minutes after all the ingredients had been placed in the drum, because this time was required for the drier mixes. Flow values on the 10-in. flow table were determined on mixes with aggregates up to $1 / 2$ in. On those with the coarser aggregate, slumps were determined.

The steel mold for casting the slab was placed over a sheet of asphalt impregnated roofing felt spread on the laboratory floor. The prepared mortar or concrete was placed in the mold, and spread and compacted with a mixing trowel. A wooden straightedge was then used to screed and further compact the material, the edges of the mold acting as a guide. Where a smooth surface could not be obtained by screeding, the surface was further smoothed with a wooden float. Final finishing with a steel trowel was done after various time intervals. Troweling was continued only long enough in each case to produce a smooth surface.

The specimens were kept in the molds in the laboratory for 24 hours. After this period the specimens not damp-cured were air-stored in the same laboratory until tested. The damp-cured slabs were placed in the damp room maintained at $70^{\circ} \pm 2^{\circ} \mathrm{F}$ and 95-percent or greater relative humidity. After damp-curing, these were also air-stored in the laboratory. All slabs except those made with high-early-strength cements were at least 28 days old when tested

The materials, mix proportions, methods of finishing, and the curing treatments are given in table 2 . 
TABLE 2.-Test results

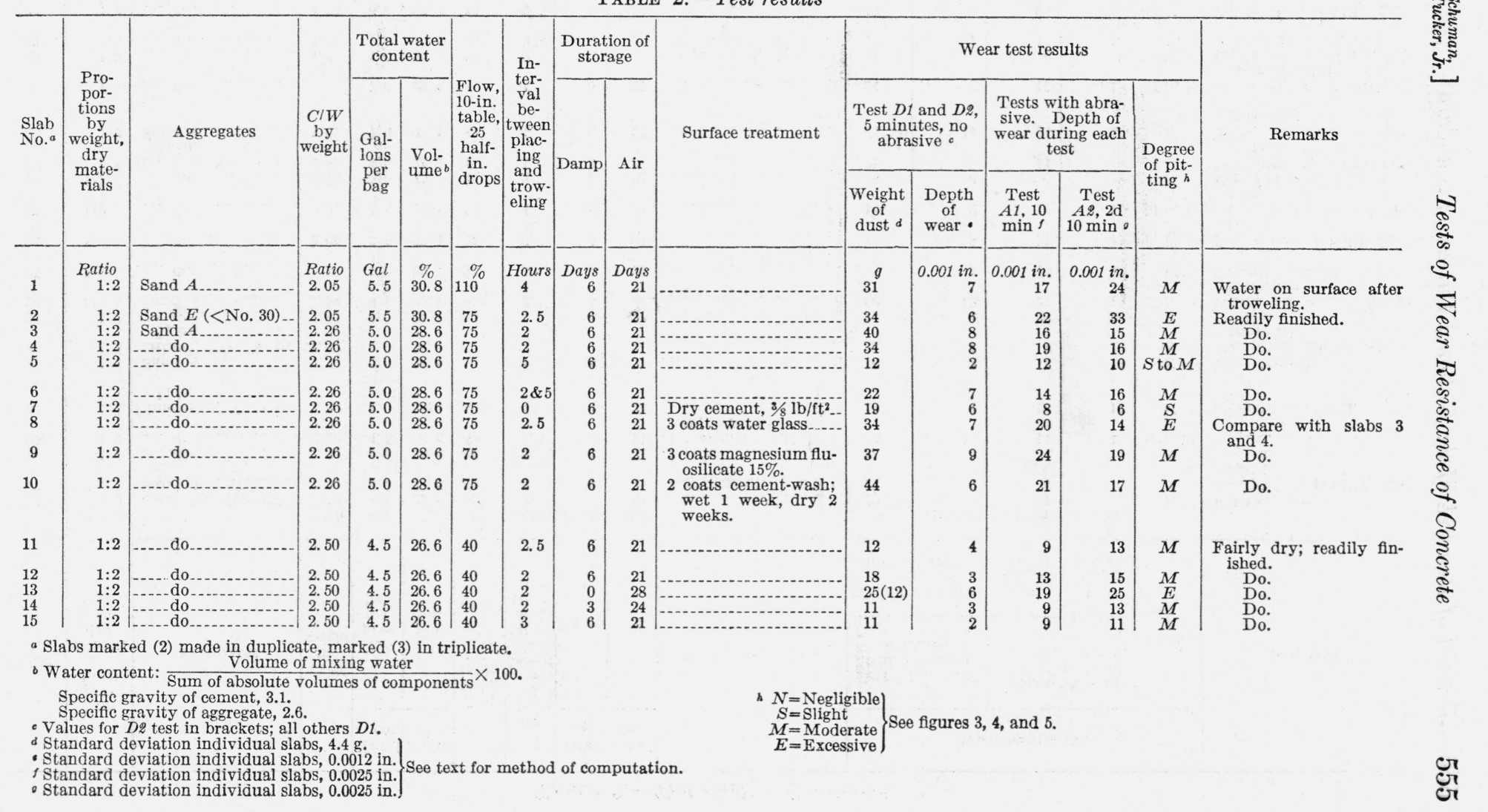


TABLe 2.-Test results-Continued

\begin{tabular}{|c|c|c|c|c|c|c|c|c|c|c|c|c|c|c|c|c|}
\hline \multirow{3}{*}{$\begin{array}{l}\text { Slab } \\
\text { No. }\end{array}$} & \multirow{3}{*}{$\begin{array}{c}\text { Pro- } \\
\text { por- } \\
\text { tions } \\
\text { by } \\
\text { weight, } \\
\text { dry } \\
\text { mate- } \\
\text { rials }\end{array}$} & \multirow{3}{*}{ Aggregates } & \multirow{3}{*}{$\begin{array}{c}C / W \\
\text { by } \\
\text { weight }\end{array}$} & \multicolumn{2}{|c|}{$\begin{array}{l}\text { Total water } \\
\text { content }\end{array}$} & \multirow{3}{*}{$\begin{array}{c}\text { Flow, } \\
10 \text {-in. } \\
\text { table, } \\
25 \\
\text { half- } \\
\text { in. } \\
\text { drops }\end{array}$} & \multirow{3}{*}{\begin{tabular}{|c} 
In- \\
ter- \\
val \\
be- \\
tween \\
plac- \\
ing \\
and \\
trow- \\
eling
\end{tabular}} & \multicolumn{2}{|c|}{$\begin{array}{c}\text { Duration of } \\
\text { storage }\end{array}$} & \multicolumn{6}{|c|}{ Wear test results } & \multirow{3}{*}{ Remarks } \\
\hline & & & & \multirow{2}{*}{$\begin{array}{l}\text { Gal- } \\
\text { lons } \\
\text { per } \\
\text { bag }\end{array}$} & \multirow{2}{*}{$\begin{array}{l}\text { Vol- } \\
\text { ume }\end{array}$} & & & \multirow[t]{2}{*}{ Damp } & \multirow{2}{*}{ Air } & \multirow[t]{2}{*}{ Surface treatment } & \multicolumn{2}{|c|}{$\begin{array}{c}\text { Test } D 1 \text { and } D 2, \\
5 \text { minutes, no } \\
\text { abrasive }\end{array}$} & \multicolumn{2}{|c|}{$\begin{array}{l}\text { Tests with abra- } \\
\text { sive. Depth of } \\
\text { wear during each } \\
\text { test }\end{array}$} & \multirow{2}{*}{$\begin{array}{l}\text { Degree } \\
\text { of pit- } \\
\text { ting }\end{array}$} & \\
\hline & & & & & & & & & & & $\begin{array}{c}\text { Weight } \\
\text { of } \\
\text { dust }\end{array}$ & $\begin{array}{l}\text { Depth } \\
\text { of } \\
\text { wear }\end{array}$ & $\begin{array}{c}\text { Test } \\
\text { min } \\
\text { min }\end{array}$ & $\begin{array}{c}\text { Test } \\
\text { A2,2d } \\
10 \mathrm{~min}\end{array}$ & & \\
\hline 16 & $\begin{array}{r}\text { Ratio } \\
1: 2\end{array}$ & Sand $D(>$ No. 16) .. & $\begin{array}{l}\text { Ratio } \\
\text { 2.50 }\end{array}$ & $\begin{array}{r}\text { Gal } \\
4.5\end{array}$ & $\begin{array}{l}\% \\
26.6\end{array}$ & $\begin{array}{l}\% \\
80^{\circ}\end{array}$ & $\begin{array}{c}\text { Hours } \\
2\end{array}$ & \begin{tabular}{|r|} 
Days \\
6
\end{tabular} & $\begin{array}{r}\text { Days } \\
21\end{array}$ & & $23^{g}$ & $\begin{array}{r}0.001 \mathrm{in} . \\
3\end{array}$ & $0.001 \mathrm{in.}$ & $\begin{array}{r}0.001 \mathrm{in} . \\
7\end{array}$ & $S$ & Fairly wet mix. \\
\hline 17 & & Sand $A$ & 2.50 & 4.5 & 26.6 & 40 & 0 & 6 & 21 & Dry cement, $1 / 4 \mathrm{lb} / \mathrm{ft}^{2}$ & 20 & & & & $\tilde{S}$ & $\begin{array}{l}\text { Fairly dry; readily fin- } \\
\text { ished. }\end{array}$ \\
\hline $\begin{array}{l}18 \\
19\end{array}$ & $\begin{array}{l}1: 2 \\
1: 2\end{array}$ & $\mid$\begin{tabular}{l}
$\mid-1$ \\
\hdashline do
\end{tabular} & $\begin{array}{l}2.50 \\
2.50\end{array}$ & $\begin{array}{l}4.5 \\
4.5\end{array}$ & $\begin{array}{l}26.6 \\
26.6\end{array}$ & $\begin{array}{l}40 \\
40\end{array}$ & $\begin{array}{l}2 \\
1.5\end{array}$ & $\begin{array}{l}6 \\
6\end{array}$ & $\begin{array}{l}21 \\
21\end{array}$ & i: 1 cement; sic No. & $\begin{array}{l}11 \\
56\end{array}$ & $\begin{array}{r}3 \\
13\end{array}$ & $\begin{array}{r}4 \\
11\end{array}$ & $\begin{array}{r}7 \\
13\end{array}$ & $\stackrel{S}{S}$ to $M$ & $\begin{array}{l}\text { Do. } \\
\text { Do. }\end{array}$ \\
\hline 20 & $1: 2$ & $\begin{array}{l}\text { Limestone, No. } 8 \text { to } \\
1 / 6 \text { in. }\end{array}$ & 2. 50 & 4.5 & 26.6 & 40 & 4 & 6 & 21 & 00,7210110 & 24 & 7 & 12 & 14 & $M$ & Difficult to trowel. \\
\hline 21 & $1: 2$ & Sand $A$ & 2.82 & 4.0 & 24.4 & 10 & 1 & 6 & 21 & & 6 & 3 & 5 & 6 & $S$ & Very dry mix. \\
\hline 22 & $1: 2$ & $\begin{array}{l}\text { Gravel, No. } 8 \text { to } \\
1 / 4 \text { in. }\end{array}$ & 2.82 & 4.0 & 24.4 & 30 & 2 & 6 & 21 & $\cdots$ & 21 & 7 & 7 & 10 & $S$ & $\begin{array}{l}\text { Somewhat sticky when } \\
\text { troweled. }\end{array}$ \\
\hline $\begin{array}{l}23 \\
24\end{array}$ & $\begin{array}{r}1: 2 \\
1: 2.5\end{array}$ & Sand $A$ & $\begin{array}{l}2.82 \\
1.88\end{array}$ & $\begin{array}{l}4.0 \\
6.0\end{array}$ & $\begin{array}{l}24.4 \\
29.1\end{array}$ & $\begin{array}{l}30 \\
95\end{array}$ & $\begin{array}{l}3 \\
3.5\end{array}$ & $\begin{array}{l}6 \\
6\end{array}$ & $\begin{array}{l}21 \\
21\end{array}$ & & $\begin{array}{l}14 \\
62\end{array}$ & $\begin{array}{r}4 \\
12\end{array}$ & $\begin{array}{l}10 \\
27\end{array}$ & $\begin{array}{l}10 \\
26\end{array}$ & $\underset{E}{S}$ & $\begin{array}{l}\text { Do. } \\
\text { Water on surface after } \\
\text { troweling. }\end{array}$ \\
\hline 25 & $1: 2.5$ & do & 1.88 & 6.0 & 29.1 & 95 & 5 & 6 & 21 & & 22 & 3 & 16 & 13 & $M$ & Readily finished. \\
\hline 26 & $1: 2.5$ & -....do do................. & 2. 05 & 5.5 & 27.4 & 55 & 2.5 & 6 & 21 & & 48 & 8 & 21 & 21 & $E$ & Fairly dry; readily fin- \\
\hline 27 & $1: 2.5$ & .....do do................. & 2.05 & 5.5 & 27.4 & 55 & 4 & 6 & 21 & & 10 & 2 & 12 & 16 & $M$ to $E$ & Difficult to trowel at \\
\hline $\begin{array}{l}28 \\
29\end{array}$ & $\begin{array}{l}1: 2.5 \\
1: 2.5\end{array}$ & Gravel, No. 8 to & $\begin{array}{l}2.26 \\
2.82\end{array}$ & $\begin{array}{l}5.0 \\
4.0\end{array}$ & $\begin{array}{l}25.5 \\
21.6\end{array}$ & $\begin{array}{l}35 \\
45\end{array}$ & $\begin{array}{l}2 \\
1.5\end{array}$ & $\begin{array}{l}6 \\
6\end{array}$ & $\begin{array}{l}21 \\
21\end{array}$ & & $\begin{array}{r}81 \\
21\end{array}$ & $\begin{array}{l}6 \\
6\end{array}$ & $\begin{array}{r}6 \\
14\end{array}$ & $\begin{array}{l}9 \\
8\end{array}$ & $\underset{S}{S}$ & $\begin{array}{l}\text { Very dry mix. } \\
\text { Difficulty in smoothing }\end{array}$ \\
\hline 30 & $1: 3$ & $\begin{array}{l}1 / 4 \text { in. } \\
\text { Sand } A\end{array}$ & 1.61 & 7.0 & 29.4 & 85 & 4 & 6 & 21 & & $74(30)$ & 14 & 33 & 25 & $E$ & $\begin{array}{l}\text { surface. } \\
\text { Water on surface after } \\
\text { troweling. }\end{array}$ \\
\hline 31 & $1: 3$ & -...do do............. & 1.61 & 7.0 & 29.4 & 85 & 5 & 6 & 21 & 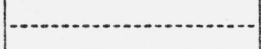 & 24 & 6 & 17 & 19 & $M$ to $E$ & $\begin{array}{l}\text { Some water on surface } \\
\text { after troweling. }\end{array}$ \\
\hline $\begin{array}{l}32 \\
33\end{array}$ & $\begin{array}{l}1: 3 \\
1: 3\end{array}$ & -do do & $\begin{array}{l}1.61 \\
1.61\end{array}$ & $\begin{array}{l}7.0 \\
7.0\end{array}$ & $\begin{array}{l}29.4 \\
29.4\end{array}$ & $\begin{array}{l}85 \\
85\end{array}$ & $\begin{array}{l}2 \\
3\end{array}$ & $\begin{array}{l}6 \\
6\end{array}$ & $\stackrel{21}{21}$ & $\begin{array}{l}\text { Dry cement, } 11 / 4 \mathrm{lb} / \mathrm{ft} \text {. } \\
1: 1 \text { cement:sand, } 1 / 2 \\
\mathrm{lb} / \mathrm{ft}^{3}\end{array}$ & $\begin{array}{l}11 \\
17\end{array}$ & $\begin{array}{l}2 \\
7\end{array}$ & $\begin{array}{r}8 \\
15\end{array}$ & $\begin{array}{r}9 \\
16\end{array}$ & $\begin{array}{l}N \\
S\end{array}$ & $\begin{array}{l}\text { Badly crazed surface. } \\
\text { Readily finished. }\end{array}$ \\
\hline 34 & $1: 3$ & 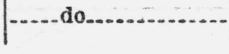 & 1. 73 & 6.5 & 27.9 & 60 & 2.5 & 6 & 21 & 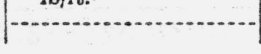 & 40 & 9 & 29 & 25 & $E$ & $\begin{array}{l}\text { Fairly dry; readily fin- } \\
\text { ished. }\end{array}$ \\
\hline
\end{tabular}



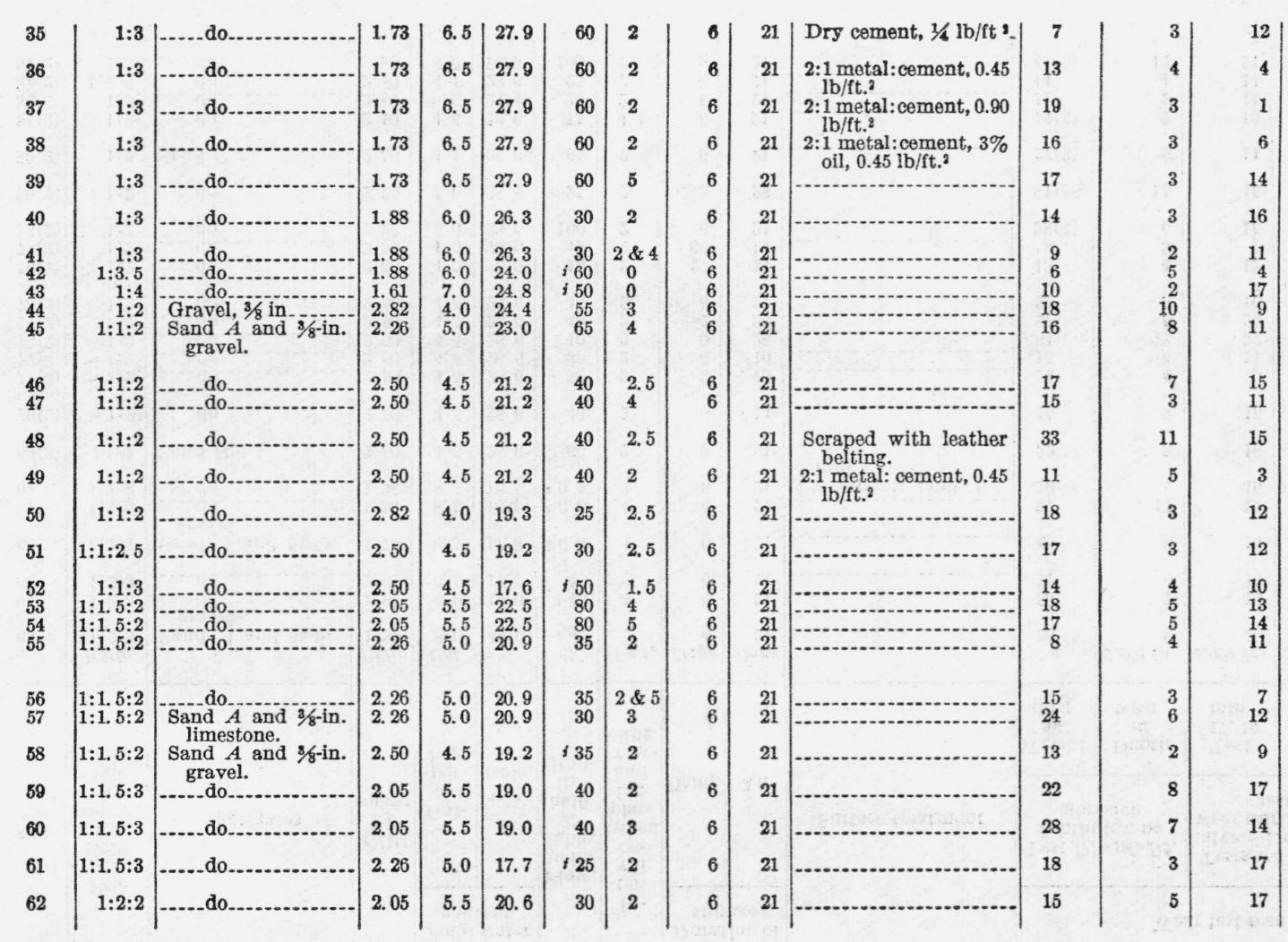
\begin{tabular}{r|r|lr}
16 & $M$ & Do. & Do. \\
3 & $M$ & Low \\
2 & $N$ & Lowest wear all slabs. & \\
12 & $E$ & Oil causes pitting. &
\end{tabular}
$16 M$ to $E$ Fairly dry; readily fin-
$18 M$ Dry; somewhat difficult
$15 \quad M$ Dry mix.
$17 \quad E$ Very dry mix.
$\begin{array}{lll} & & \\ 14 & S & \text { Difficult to trowel. }\end{array}$
Fairly dry when finished.
Somewhat wet.
Sinewhat wet; difficult
to finish.
Difficult to trowel.
Dry mix.
Difficult to trowel; un- dersanded.
Somewhat wet.
Do. eled.
Do.
Fairly dry; readily fin. Fairly dry; easily fin- ished.
slightly wet; easily fin. Do.
Dry mix; diffeult to trowel. slightly wet; readily fin
ished.


TABLE 2.-Test results-Continued

\begin{tabular}{|c|c|c|c|c|c|c|c|c|c|c|c|c|c|c|c|c|}
\hline \multirow{3}{*}{$\begin{array}{l}\text { Slab } \\
\text { No. }\end{array}$} & \multirow{3}{*}{$\begin{array}{c}\text { Pro- } \\
\text { por- } \\
\text { tions } \\
\text { by } \\
\text { weight, } \\
\text { dry } \\
\text { mate- } \\
\text { rials }\end{array}$} & \multirow{3}{*}{ Aggregates } & \multirow{3}{*}{$\mid \begin{array}{c}C / W \\
\text { by } \\
\text { weight } \\
\end{array}$} & \multicolumn{2}{|c|}{$\begin{array}{l}\text { Total water } \\
\text { content }\end{array}$} & \multirow{3}{*}{\begin{tabular}{|c|} 
\\
Flow, \\
10 -in. \\
table, \\
25 \\
half- \\
in. \\
drops
\end{tabular}} & \multirow{3}{*}{\begin{tabular}{|c|} 
\\
In- \\
ter- \\
val \\
be- \\
tween \\
plac- \\
ing \\
and \\
trow- \\
eling
\end{tabular}} & \multicolumn{2}{|c|}{$\begin{array}{c}\text { Duration of } \\
\text { storage }\end{array}$} & \multicolumn{6}{|c|}{ Wear test results } & \multirow{3}{*}{ Remarks } \\
\hline & & & & \multirow{2}{*}{$\begin{array}{l}\text { Gal- } \\
\text { lons } \\
\text { per } \\
\text { bag }\end{array}$} & \multirow{2}{*}{$\begin{array}{l}\text { Vol- } \\
\text { ume }\end{array}$} & & & \multirow{2}{*}{ Damp } & \multirow{2}{*}{ Air } & \multirow[t]{2}{*}{ Surface treatment } & \multicolumn{2}{|c|}{$\begin{array}{c}\text { Test } D 1 \text { and } D 2, \\
5 \text { minutes, no } \\
\text { abrasive }\end{array}$} & \multicolumn{2}{|c|}{$\begin{array}{c}\text { Tests with abra- } \\
\text { sive. Depth of } \\
\text { wear during each } \\
\text { test }\end{array}$} & \multirow{2}{*}{$\begin{array}{l}\text { Degree } \\
\text { of pit- } \\
\text { ting }\end{array}$} & \\
\hline & & & & & & & & & & & $\begin{array}{c}\text { Weight } \\
\text { of } \\
\text { dust }\end{array}$ & $\begin{array}{c}\text { Depth } \\
\text { of } \\
\text { wear }\end{array}$ & $\begin{array}{l}\text { Test } \\
\text { A1, } 10 \\
\text { min }\end{array}$ & $\begin{array}{l}\text { Test } \\
A 2,2 \mathrm{~d} \\
10 \mathrm{~min}\end{array}$ & & \\
\hline 63 & $\begin{array}{l}\text { Ratio } \\
1: 2: 3\end{array}$ & $\begin{array}{l}\text { Sand } A \text { and } 3 / 8-i n . \\
\text { gravel- }\end{array}$ & $\begin{array}{c}\text { Ratio } \\
1.88\end{array}$ & $\begin{array}{l}\text { Gal } \\
6.0\end{array}$ & $\begin{array}{l}\% \\
19.0\end{array}$ & $\begin{array}{c}\% \\
i 45\end{array}$ & $\begin{array}{c}\text { Hours } \\
2\end{array}$ & $\mid \begin{array}{c}\text { Days } \\
6\end{array}$ & $\begin{array}{r}\text { Days } \\
21\end{array}$ & 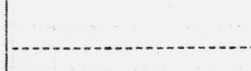 & $25^{g}$ & $0.001 \mathrm{in}$ & $\begin{array}{r}0.001 \text { in. } \\
20\end{array}$ & $\begin{array}{r}0.001 \text { in } \\
12\end{array}$ & $M$ & $\begin{array}{l}\text { Fairly dry; somewhat } \\
\text { difficult to finish. }\end{array}$ \\
\hline $\begin{array}{l}64 \\
65\end{array}$ & $\begin{array}{l}1: 2: 3 \\
1: 2: 3\end{array}$ & 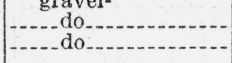 & $\begin{array}{l}1.88 \\
2.05\end{array}$ & $\begin{array}{l}6.0 \\
5.5\end{array}$ & $\begin{array}{l}\text { 19. } 0 \\
17.7\end{array}$ & $\begin{array}{r}j 45 \\
40\end{array}$ & $\begin{array}{l}3 \\
2\end{array}$ & $\begin{array}{l}6 \\
6\end{array}$ & $\begin{array}{l}21 \\
21\end{array}$ & & $\begin{array}{l}33 \\
17\end{array}$ & $\begin{array}{l}7 \\
6\end{array}$ & $\begin{array}{l}13 \\
13\end{array}$ & $\begin{array}{l}10 \\
12\end{array}$ & $\stackrel{M}{S}$ & $\begin{array}{l}\text { Do. } \\
\text { Do. }\end{array}$ \\
\hline 66 & $1: 2: 4$ & $\begin{array}{l}\text { Sand } A \text { and } 3 / 4 \text {-in. } \\
\text { gravel. }\end{array}$ & 1. 73 & 6.5 & 17.8 & ${ }^{k} 0.3$ & 4 & 6 & 21 & & 48 & 9 & 16 & 12 & $S$ & $\begin{array}{l}\text { Considerable floating re- } \\
\text { quired. }\end{array}$ \\
\hline $\begin{array}{l}67 \\
68\end{array}$ & $\begin{array}{l}1: 3: 6 \\
1: 3: 6\end{array}$ & - do do & $\begin{array}{l}\text { 1. } 33 \\
\text { 1. } 33\end{array}$ & $\begin{array}{l}8.5 \\
8.5\end{array}$ & $\begin{array}{l}16.5 \\
16.5\end{array}$ & $\begin{array}{l}{ }_{k}^{k} 0.5 \\
{ }^{k} 0.5\end{array}$ & $\begin{array}{l}4 \\
3\end{array}$ & $\begin{array}{l}6 \\
6\end{array}$ & $\begin{array}{l}21 \\
21\end{array}$ & $\begin{array}{l}1: 2 \text { cement: sand, } 0.5 \\
\mathrm{lb} / \mathrm{ft}^{2}\end{array}$ & $\begin{array}{l}57 \\
19\end{array}$ & $\begin{array}{r}14 \\
4\end{array}$ & $\begin{array}{l}22 \\
16\end{array}$ & $\begin{array}{l}13 \\
15\end{array}$ & $\underset{S}{S}$ & $\begin{array}{l}\text { Do. } \\
\text { Dust coat aids finishing. }\end{array}$ \\
\hline $69(3)$ & $l 1: 2$ & Sand $B \ldots$ & 2. 50 & 4. 5 & 26.6 & 60 & 2 & 6 & 21 & & 22 & 3 & 18 & 13 & $M$ & $\begin{array}{l}\text { Fairly dry; readily fin- } \\
\text { ished. }\end{array}$ \\
\hline $70(2)$ & $m 1: 2$ & ...... do & 2.50 & 4.5 & 26.6 & 40 & 2 & 0 & 14 & & 16 & 3 & 15 & 13 & $M$ & Do. \\
\hline $\begin{array}{l}71(3) \\
72(3) \\
73(2) \\
74(2) \\
75(2)\end{array}$ & $\begin{array}{rl}m & 1: 2 \\
m & 1: 2 \\
1: 2 \\
1: 2 \\
\\
1: 2\end{array}$ & 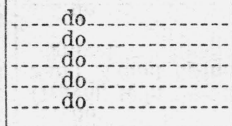 & $\begin{array}{l}\text { 2. } 50 \\
\text { 2. } 50 \\
2.50 \\
2.50 \\
2.50\end{array}$ & $\begin{array}{l}\text { 4. } 5 \\
\text { 4. } 5 \\
\text { 4. } 5 \\
\text { 4. } 5 \\
\text { 4. } 5\end{array}$ & $\begin{array}{l}26.6 \\
26.6 \\
26.6 \\
26.6 \\
26.6\end{array}$ & $\begin{array}{l}30 \\
35 \\
40 \\
40 \\
45\end{array}$ & $\begin{array}{l}2 \\
2 \\
2 \\
2 \\
2\end{array}$ & $\begin{array}{l}2 \\
6 \\
0 \\
2 \\
6\end{array}$ & $\begin{array}{l}10 \\
10 \\
28 \\
25 \\
21\end{array}$ & (n) & $\begin{array}{l}14 \\
15 \\
64 \\
27 \\
25\end{array}$ & $\begin{array}{r}4 \\
4 \\
13 \\
5 \\
5\end{array}$ & $\begin{array}{l}10 \\
11 \\
32 \\
20 \\
16\end{array}$ & $\begin{array}{l}13 \\
11 \\
27 \\
19 \\
12\end{array}$ & $\begin{array}{l}M \\
M \\
E \\
M \\
M\end{array}$ & $\begin{array}{l}\text { Do. } \\
\text { Do. } \\
\text { Do. } \\
\text { Do. } \\
\text { Compare with No. } 96 .\end{array}$ \\
\hline $\begin{array}{l}76(2) \\
77(2) \\
78(2)\end{array}$ & $\begin{array}{l}1: 2 \\
1: 2 \\
1: 2\end{array}$ & \begin{tabular}{|c|} 
do \\
do
\end{tabular} & $\begin{array}{l}2.50 \\
2.50 \\
2.26\end{array}$ & $\begin{array}{l}4.5 \\
\text { 4. } 5 \\
5.0\end{array}$ & $\begin{array}{l}26.6 \\
26.6 \\
28.6\end{array}$ & $\begin{array}{r}45 \\
45 \\
100\end{array}$ & $\begin{array}{l}2 \\
2 \\
2\end{array}$ & $\begin{array}{r}27 \\
60 \\
6\end{array}$ & $\begin{array}{l}14 \\
14 \\
21\end{array}$ & & $\begin{array}{l}13 \\
8 \\
28(4)\end{array}$ & $\begin{array}{l}4 \\
3 \\
6\end{array}$ & $\begin{array}{r}12 \\
7 \\
15\end{array}$ & $\begin{array}{r}11 \\
8 \\
11\end{array}$ & $\stackrel{S}{S}$ & $\begin{array}{l}\text { Compare with No. } 75 . \\
\text { Do. } \\
\text { Compare with No. } 97 \text {, }\end{array}$ \\
\hline $79(2)$ & $1: 2$ & .....do do & 2. 26 & 5.0 & 28. 6 & 95 & 2 & 0 & 28 & & $81(56)$ & 15 & 45 & 26 & $E$ & Compare with No. 100 , \\
\hline $80(2)$ & $1: 2$ & Sand $C_{\ldots} \ldots \ldots$ & 2.50 & 4. 5 & 26.6 & 85 & 2 & 6 & 21 & & $27(3)$ & 6 & 14 & 11 & $M$ & Somewhat wet. \\
\hline $\begin{array}{l}81(2) \\
82(2) \\
83(2) \\
84(2)\end{array}$ & $\begin{array}{r}1: 2 \\
1: 2 \\
1: 2.5 \\
1: 3\end{array}$ & \begin{tabular}{|c|} 
do \\
do
\end{tabular} & $\begin{array}{l}2.50 \\
2.82 \\
2.50 \\
1.88\end{array}$ & $\begin{array}{l}4.5 \\
4.0 \\
4.5 \\
6.0\end{array}$ & $\begin{array}{l}26.6 \\
24.4 \\
23.6 \\
26.3\end{array}$ & $\begin{array}{r}75 \\
20 \\
25 \\
100\end{array}$ & $\begin{array}{l}4.5 \\
2 \\
2 \\
5\end{array}$ & $\begin{array}{l}6 \\
6 \\
6 \\
6\end{array}$ & $\begin{array}{l}21 \\
21 \\
21 \\
21\end{array}$ & & $\begin{array}{l}10(3) \\
11 \\
14 \\
41(6)\end{array}$ & $\begin{array}{r}2 \\
2 \\
4 \\
12\end{array}$ & $\begin{array}{l}10 \\
11 \\
14 \\
21\end{array}$ & $\begin{array}{l}10 \\
12 \\
14 \\
16\end{array}$ & $\begin{array}{l}\stackrel{S}{M} \\
\stackrel{M}{M}\end{array}$ & $\begin{array}{l}\text { Do. } \\
\text { Very dry mix. } \\
\text { Do. wet; compare } \\
\text { Rather wet } \\
\text { with No. } 40 .\end{array}$ \\
\hline
\end{tabular}




\begin{tabular}{|c|c|c|c|c|c|c|c|c|c|c|c|c|c|c|c|c|}
\hline $85(2)$ & $1: 3$ & |-_...do-_._. & 2. 05 & 5.5 & 23.7 & 30 & 2 & 6 & 21 & & 17 & 5 & 18 & 16 & $M$ & Very dry mix. \\
\hline $86(2)$ & $1: 1: 2$ & Sand $C$ and $3 / 8$-in. & 2. 50 & 4.5 & 21.2 & 40 & 2 & 6 & 21 & & 16 & 6 & 15 & 10 & $S$ & Dry; difficult to trowel; \\
\hline $\begin{array}{l}87(2) \\
88(2)\end{array}$ & $\begin{array}{r}1: 1: 2 \\
1: 1.5: 2\end{array}$ & \begin{tabular}{|} 
gravel. \\
\hdashline do \\
do-_and \\
\end{tabular} & $\begin{array}{l}2.26 \\
2.26\end{array}$ & $\begin{array}{l}5.0 \\
5.0\end{array}$ & $\begin{array}{l}23.0 \\
20.9\end{array}$ & $\begin{array}{l}85 \\
40\end{array}$ & $\begin{array}{lll}2 & \& & 5 \\
2 & \& & 5\end{array}$ & $\begin{array}{l}6 \\
6\end{array}$ & $\begin{array}{l}21 \\
21\end{array}$ & & $\begin{array}{l}17 \\
16\end{array}$ & $\begin{array}{l}4 \\
4\end{array}$ & $\begin{array}{l}19 \\
14\end{array}$ & $\begin{array}{r}11 \\
9\end{array}$ & $\stackrel{S}{S}$ & $\begin{array}{l}\text { Wet mix. } \\
\text { Fairly dry; difficult to }\end{array}$ \\
\hline $\begin{array}{l}89(2) \\
90(2)\end{array}$ & $\begin{array}{l}1: 1.5: 2 \\
1: 1.5: 2\end{array}$ & do... do & $\begin{array}{l}2.05 \\
2.05\end{array}$ & $\begin{array}{l}5.5 \\
5.5\end{array}$ & $\begin{array}{l}22.5 \\
22.5\end{array}$ & $\begin{array}{l}80 \\
85\end{array}$ & $2 \& 5$ & $\begin{array}{l}6 \\
6\end{array}$ & $\begin{array}{l}21 \\
21\end{array}$ & 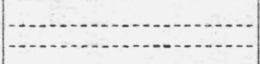 & $\begin{array}{l}13 \\
17(1)\end{array}$ & $\begin{array}{l}4 \\
5\end{array}$ & $\begin{array}{l}19 \\
20\end{array}$ & $\begin{array}{l}12 \\
11\end{array}$ & $\underset{S}{S}$ & $\begin{array}{l}\text { Fairly wet. } \\
\text { Do. }\end{array}$ \\
\hline $91(2)$ & $1: 2: 2$ & .....do-.... & 2.05 & 5.5 & 20.6 & 60 & 2 & 6 & 21 & & 45 & 12 & 18 & 14 & $S$ & $\begin{array}{c}\text { Difficult to float and } \\
\text { trowel. }\end{array}$ \\
\hline 92 & $1: 2: 3$ & $\begin{array}{l}\text { Sand } C \text { and } 3 / 4 \text {-in. } \\
\text { gravel. }\end{array}$ & 1.88 & 6.0 & 19.0 & ${ }^{k} 1.1$ & $2 \& 5$ & 6 & 21 & & $25(1)$ & 4 & 20 & 12 & $S$ & $\begin{array}{l}\text { Fairly wet; easily placed } \\
\text { and troweled. }\end{array}$ \\
\hline 93 & $1: 2: 4$ & do & 1. 73 & 6.5 & 17.8 & ${ }^{k} 0.5$ & 2 & 6 & 21 & & $69(2)$ & 18 & 24 & 12 & $S$ & $\begin{array}{l}\text { Difficult to trowel; wet } \\
\text { surface. }\end{array}$ \\
\hline 94 & $1: 1.5: 2$ & $\begin{array}{l}\text { Sand } F \text { and } 1 / 2 \text {-in. } \\
\text { traprock. }\end{array}$ & 2.05 & 5.5 & 22.5 & 30 & 4 & 6 & 21 & & 30 & 8 & 17 & 10 & $S$ & Do. \\
\hline $95(2)$ & $1: 2: 2$ & - do do............ & 1.88 & 6.0 & 22.1 & 40 & 4 & 6 & 21 & & 55 & 15 & 19 & 11 & $S$ & $\begin{array}{l}\text { Difficult to finish; wet } \\
\text { surface. }\end{array}$ \\
\hline $96(2)$ & $1: 2$ & Sand $B \ldots$ & 2. 50 & 4.5 & 26.6 & 40 & 2 & 6 & 21 & $\begin{array}{l}3 \text { coats high silica } \\
\text { water glass. }\end{array}$ & 19 & 4 & 13 & 13 & $M$ & Compare with No. 75 . \\
\hline $\begin{array}{l}97(2) \\
98(2)\end{array}$ & $\begin{array}{l}1: 2 \\
1: 2\end{array}$ & (ndo......... & $\begin{array}{l}2.26 \\
2.26\end{array}$ & $\begin{array}{l}5.0 \\
5.0\end{array}$ & $\begin{array}{l}28.6 \\
28.6\end{array}$ & $\begin{array}{l}90 \\
95\end{array}$ & $\begin{array}{l}2 \\
2\end{array}$ & $\begin{array}{l}6 \\
6\end{array}$ & $\begin{array}{l}21 \\
21\end{array}$ & $\begin{array}{l}3 \text { coats low silica water } \\
\text { glass. }\end{array}$ & $\begin{array}{l}27(5) \\
27(4)\end{array}$ & $\begin{array}{l}6 \\
8\end{array}$ & $\begin{array}{l}20 \\
16\end{array}$ & $\begin{array}{l}18 \\
16\end{array}$ & $\begin{array}{l}M \\
M\end{array}$ & $\begin{array}{l}\text { Compare with No. } 78 \text {. } \\
\text { Do. }\end{array}$ \\
\hline $99(2)$ & $1: 2$ & ....... do & 2. 26 & 5. 0 & 28.6 & 85 & 2 & 6 & 21 & $\begin{array}{l}3 \text { coats magnesium } \\
\text { fluosilicate. }\end{array}$ & $27(5)$ & 6 & 15 & 13 & $M$ & Do. \\
\hline $100(2)$ & $1: 2$ & ......do... & 2. 26 & 5.0 & 28.6 & 100 & 2 & 0 & 28 & 3 coats water & $79(17)$ & 15 & 30 & 22 & $M$ & Compare with No. 79. \\
\hline $101(2)$ & $1: 2$ & do & 2. 26 & 5.0 & 28.6 & 95 & 2 & 0 & 28 & 3 coats high silica & 66 & 14 & 32 & 31 & $E$ & Compare with No. 79 \\
\hline $102(2)$ & $1: 2$ & ........ do... & 2. 26 & 5.0 & 28.6 & 95 & 2 & 0 & 28 & 3 coats low silica water & $54(22)$ & 10 & 26 & 24 & $E$ & Do. \\
\hline $103(2)$ & $1: 2$ & ............... & 2. 26 & 5.0 & 28.6 & 80 & 2 & 0 & 28 & $\begin{array}{l}3 \text { coats magnesium } \\
\text { fluosilicate. }\end{array}$ & $53(31)$ & 10 & 30 & 30 & $E$ & Do. \\
\hline & & & & & & & & & & $\begin{array}{l}\text { Maximum } \\
\text { Minimum }\end{array}$ & $\begin{array}{c}81(56) \\
6(1)\end{array}$ & $\begin{array}{r}18 \\
2\end{array}$ & $\begin{array}{r}45 \\
1\end{array}$ & $\begin{array}{r}33 \\
2\end{array}$ & & \\
\hline
\end{tabular}

i. Flow on "dry" side: See text.

${ }^{k}$ Slump, inches.

w High-early-strength cement. 
The liquid "hardeners" were applied to 15 slabs of a 1:2 mortar when the slabs were 28 days old. The water-glass solutions were applied in three coats, on successive days, each solution being applied liberally with a brush. The slabs were not scrubbed with water between coats, as usually recommended [1], since such scrubbing might have the effect of additional damp-curing. After the last coat, the specimens were air-stored 2 weeks before testing.

The magnesium fluosilicate solutions were also applied in three coats. The 15-percent solution was applied without dilution. The 22-percent solution was diluted with two volumes of water for the first coat, with one volume for the second coat, and with one-half volume for the third coat. The second and third coats of the fluosilicate solutions were absorbed better than the corresponding coats of water-glass solutions, as might be expected.

To determine any possible effect of the water in the solutions on specimens not previously damp-cured, two slabs of mix 100 were given three surface applications of water, in amounts about equal to the amounts of magnesium fluosilicate or of water-glass solutions applied to the other slabs.

Most of the specimens with sand $C$ and those made for comparing cements, curing periods, and liquid "hardener" treatments were made in duplicate or in triplicate, as indicated in table 2. It was not considered necessary to make duplicate or triplicate specimens in other cases.

\section{TEST METHODS}

Preliminary tests had shown that a 5-minute test without abrasive might give information of value, since the surface layer or "skin" was often more easily abraded than the subsurface. Consequently, all of the test slabs were first abraded by this method, which is designated test D1. The loosened dust was brushed off and weighed, and the depth of wear measured at eight equally spaced positions.

Two 10-minute tests, designated $A 1$ and $A 2$, were then made on the same section of the slab, using abrasive; and the depth of wear for each test was measured.

Twenty-eight slabs were given a further 5-minute test without abrasive, which is designated $D 2$, on the area already abraded in tests $D 1, A 1$, and $A 2$; and the weight of dust was determined as in test $D 1$.

The degrees of pitting (table 2) were estimated by inspection.

The amount of dust removed in each of six successive half-minute tests from a section of the surface not abraded in the previous tests was determined for the slabs treated with liquid hardeners and for the corresponding untreated specimens (slabs 78, 79, and 97 to 103).

The reproducibility of results in table 2 is illustrated by like slabs 3 and 4 and also slabs 11 and 12 . Individual test data are not given on the remainder of the slabs made in duplicate or triplicate. To furnish information on the reproducibility of the results obtained upon like slabs, the dispersion for the individual slab was measured, as expressed by the standard deviation, and computed from the formula:

$$
\sigma=\sqrt{\frac{\Sigma d^{2}}{N-n}},
$$


where $d$ is the deviation of the average value for a slab from the average for a like group ( 2 or 3 slabs in this case), $n$ is the number of groups of like slabs, and $N$ is the total number of slabs in these groups.

The reproducibility of the results is as follows:

\begin{tabular}{|c|c|}
\hline Test & $\begin{array}{l}\text { Standard } \\
\text { deviation }\end{array}$ \\
\hline $\begin{array}{l}D 1 \text { - weight of dust in grams } \\
D 1=\text { depth of wear in } 0.001 \text { in } \\
A 1 \text { - depth of wear in } 0.001 \text { in } \\
A 2\end{array}$ & $\begin{array}{l}4.4 \\
1.2 \\
2.5 \\
2.5\end{array}$ \\
\hline
\end{tabular}

Computations were made also of the deviations in the eight individual readings on a single slab. These deviations are given in the following tabulation under the head of standard deviation of the individual measurement. These computations were made for a group of 10 representative slabs:

\begin{tabular}{|c|c|c|}
\hline \multirow{2}{*}{ Test } & \multicolumn{2}{|c|}{ Standard deviation } \\
\hline & $\begin{array}{c}\text { Individual } \\
\text { measurement }\end{array}$ & Slab \\
\hline $\begin{array}{l}D 1 \text {-depth of wear in } 0.001 \text { in } \\
A 1 \text { - depth of wear in } 0.001 \text { in } \\
A 2 \text {-depth of wear in } 0.001 \text { in }\end{array}$ & $\begin{array}{l}2.5 \\
5.5 \\
6.5\end{array}$ & $\begin{array}{l}1.0 \\
2.0 \\
2.3\end{array}$ \\
\hline
\end{tabular}

In the last column of the tabulation is given the theoretical standard deviation of a single slab computed from the standard deviation of the individual measurements. This standard deviation of the single slab is the standard deviation of the individual measurement divided by the square root of the number of individual measurements made on one slab - eight in this case.

From the values of the theoretical standard deviation of the slab and the standard deviation computed for like slabs in the first tabulation, it is at once apparent that variations between different slabs are of the same magnitude as would be caused by the variations in the individual readings on a single slab and that results on duplicate slabs do not differ significantly from each other.

\section{TEST RESULTS}

\section{GENERAL DISCUSSION}

The weights of dust removed in test $D 1$ vary from 6 to $81 \mathrm{~g}$, and the depths of wear in the same test from 0.002 to 0.018 in. In test $A 1$ the depths of wear varied from 0.001 to 0.045 in., and in test $A 2$ from 0.002 to $0.035 \mathrm{in}$. The values for test $D_{2}^{2}$ are generally considerably lower than for test $D 1$.

The relative depths of wear in tests $D 1$ and $A 1$ depend on the mix, finishing procedure, and other factors, especially surface treatment. For most of the tests, the depth of wear for test $A 1$ is from two to five times as much as for test D1. In 10 cases, however, the depth for test $A 1$ differs little from or is less than that for test $D 1$, indicating a highly resistant subsurface compared with the surface layer (for 
example, Nos. $7,17,28,37)$. Where the values for the $D 1$ tests are high, exceeding 0.011 in., indicating a comparatively poor surface layer, the subsurface may be comparatively resistant, with values in the $A 1$ test less than 0.023 in. (slabs 48, 67, 91, 95).

For most of the slabs, the wear in test $A 2$ was approximately equal to or less than that in test $A 1$; however, for a few of the slabs it was considerably higher (slabs 2, 42, 43).

\section{EFFECT OF VARIOUS FACTORS ON WEAR}

(a) WATER CONTENT AND GRADING OF AGGREGATE

Most of the mixes were moderately dry, although some were sufficiently wet to have flow values as high as 110 . As is known, decrease in water content will decrease the flow-table reading to a certain point, which we will term the minimum flow, beyond which further decrease in water content will cause increase in flow readings. Thus, two mixes with very different water contents may give equal flow readings, one of the mixes being fairly wet, the other dry. The dry mixes are indicated in table 2.

Most of the mixes used could be finished readily by hand floating and troweling. In some cases, however, difficulty was encountered especially where there appeared to be an excess of coarse aggregate or insufficient sand-for example, specimens 51 and 52 . The amount of floating and troweling necessary in such cases sometimes brought free water to the surface (slabs 93, 94, and 95). The wetter mortar mixes also showed surface water, especially if troweled too soon (slabs 1, $24,30)$. Although excess water was not the only cause of poor wear resistance, its appearance on the slabs, as noted in table 2 , usually was followed by poor wear resistance in the $D 1$ test, for example, slabs 24, $30,93,94$, and 95 .

Figure 3 shows the total depth of wear, after completion of tests $D 1, A 1$, and $A 2$, plotted for mortars and concretes of various proportions and the $C / W$ ratios in which sand $A$ or $C$, with or without $3 / 8-$ or $3 / 4$-in. gravel, was used. For any particular mix proportions the depth of wear decreases in general with increasing $C / W$ ratios. For example, the $1: 2$ mortar shows a decrease from 0.048 to 0.014 in., as the $C / W$ ratio is increased from 2.05 to 2.82 . For the slabs containing gravel there is also a decrease, though relatively smaller.

For a particular $C / W$ ratio the total depth of wear of the mortar mixes, in three out of five cases, decreases considerably as the proportion of the sand increases, in the other two cases showing little change (fig. 3). For the mixes containing gravel the depth of wear is lowest for the 1:1.5:2 mix. For both sand $C$ and sand $A$ the depth of wear increases as the gravel mixes become leaner in cement. The 1:2 mix, $C / W=2.26$, showed about twice the depth of wear obtained on the $1: 1.5: 2 \mathrm{mix}$ of the same $C / W$ ratio; however, by raising the sand-cement ratio of the former to 2.5 and keeping $C / W=2.26$, the depth of wear is decreased to about that for the latter. A similar comparison may be made between the 1:2.5 mortar and the 1:2:3 concrete for $C / W=2.05$. Here the replacement of one-half part of sand by three parts of $3 / 8$-in. gravel decreases the depth of wear by almost 40 percent, though the cement content is halved. Thus, it appears that wear resistance depends both on proportion of aggre- 
gate and on the $C / W$ ratio, and that the drier the mix, within certain limits, the higher the resistance to wear.

That the decrease in depth of wear is not due merely to the presence of coarser material, such as gravel, is shown by the fact that a $1: 2$ mortar can be made dry enough so that the depth of wear will be equal to or less than that of the mixes containing gravel. For example, the $1: 2 \mathrm{mix}, C / W=2.50$ (table 2 , slabs 11 and 12 ) gives about the same total depth of wear as some mixes with gravel, such as slabs 46,61 , and 62. A still drier 1:2 mix, $C / W=2.82$ (slab 21) gave lower total depth of wear than any other, except those with special finishes, such as slabs 36 and 49 .

For most of the mortar mixes the depths of wear in tests $A 1$ and A2 were more nearly equal than for the mixes containing coarse

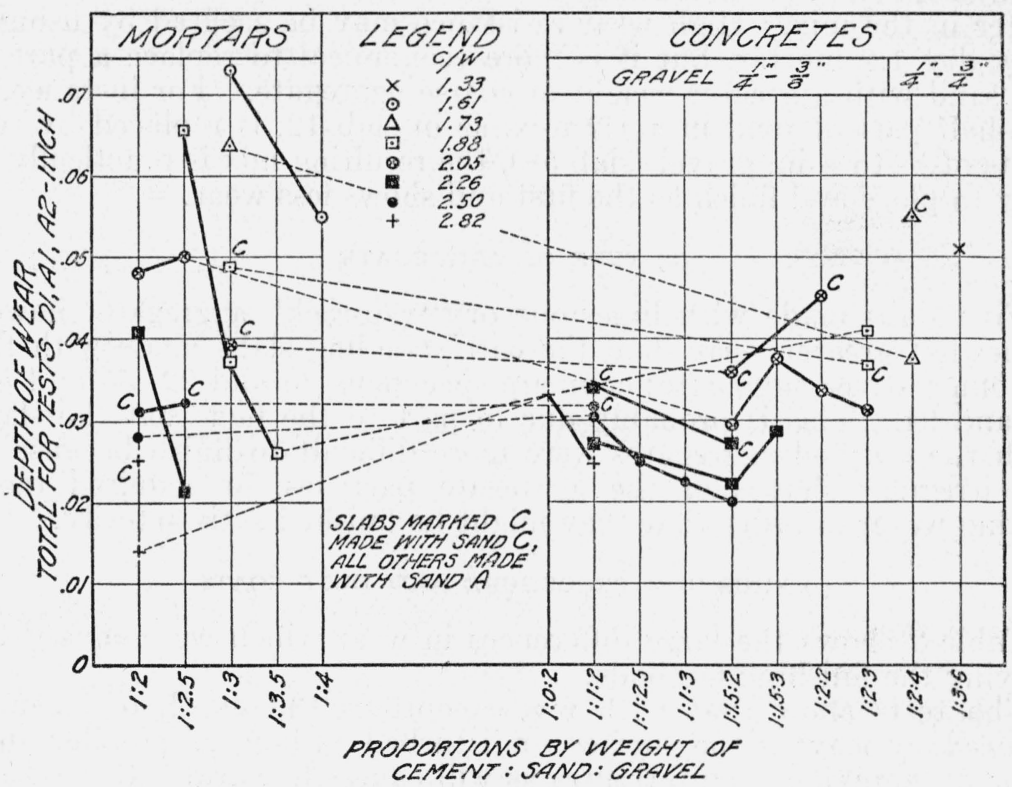

Figure 3.-Depth of wear for mixes of various proportions and $C / W$ ratios.

All slabs trowled $4 \mathrm{hr}$ or less after placing.

aggregate. For example, for slabs 75 to 78 and 80 to 85 , which do not contain coarse aggregate, the $A 2$ wear was never less than 75 percent of the $A 1$ wear, the average value being 92 percent; for slabs 86 to 95 , containing coarse aggregate, the wear in the $A 2$ test varied from 50 to 78 percent of the $A 1$ wear and averaged 61 percent.

Higher flow values were generally obtained with sand $C$ for equal $C / W$ ratios and mix proportions than with the finer sand, $A$. The effect on wear resistance of these sands is readily distinguished by use of the apparatus. The slabs with sand $C$ generally showed greater wear for equal $C / W$ ratios than those with sand $A$, as shown in figure 3. In some cases, however, there is little difference in either flow or wear, due to the use of different sands, as for the 1:1:2 mix, $C / W=2.50$ (slabs 46 and 86). The greater wear for some of the coarser sand 
mixes is probably caused by difficulty in finishing, causing water to be brought to the surface as shown by slab 91 compared with slab 62 , and slab 93 compared with slab 66 . The mixes with gravel and the coarser sand require a higher proportion of sand for proper finishing, so that a $1: 2: 3 \mathrm{mix}$ (slab 92) is preferable to a $1: 2: 4 \mathrm{mix}$ (slab 93).

The mixes with gravel only, such as a No. 8 to $1 / 4$ in. or a $1 / 4$ to $3 / 8$ in. showed comparatively little wear (slabs $22,23,29,44$ ). Slab 16, of 1:2 mortar, made with a sand all retained on the No. 16 sieve (sand $D$ ), showed very slight wear. With such an aggregate, the water content can be reduced to that corresponding to $C / W=2.82$. At the other extreme, a mortar with sand $E$ all passing the No. 30 sieve showed fairly high wear.

For minimum wear it thus appears necessary to limit the amounts both of fine and of coarse aggregate as well as to limit the amount of water in the mix. High wear resistance may be secured by using a very dry 1:2 mortar, but it is more economical to replace a part of the sand with a greater weight of coarse aggregate. For instance, if one-half part of sand in a 1:2 mix, as in slab 12, is replaced by two parts of $1 / 4$ - to $3 / 8$-in. gravel (slab 58), the resulting mix is practically as easy to place and finish as the first and shows less wear.

\section{(b) TYPE OF AGGREGATE}

Five slabs made with limestone or "trap-rock" aggregate showed somewhat greater wear than the corresponding slabs made with the Potomac River aggregate; compare specimens 20 and 22, 55 and 57, 53 and 94 . This is probably due in part to the fact that the slabs with the crushed aggregates were more difficult to finish because of the irregular shapes of the aggregate particles, or required more mixing water in order that they might be finished satisfactorily.

\section{(c) FINISHING PROCEDURE AND DUST COATS}

Table 2 shows the large differences in wear which were caused by varying the finishing methods.

The tests show that with few exceptions the depth of wear is reduced by leaving the surface undisturbed as long as possible (fig. 4) or by applying such mixtures as will cause the surface to be drier or to be richer in cement than the subsurface (fig. 5). It has already been shown that the drier mixes show the least wear. The greatest increase in wear resistance which can be brought about by delayed troweling is generally in that portion of the slab nearest the surface, as shown by results for the $D 1$ test, figure 4 . A single delayed troweling appears to be more effective than two trowelings.

It is commonly believed that "fines," brought to the surface by, troweling excessively or prematurely, are the cause of "dusting." The poor wear resistance of the wetter mixes appears more likely to be caused by water brought to the surface in finishing, since the addition of fines in the form of dust coats containing cement greatly reduces the depth of wear. The use of a dust coat also enables easier and earlier finishing in some cases, as for slab 68 compared with slab 67. The reductions in depth of wear effected by the use of dust coats are considerable in all three tests, as seen in figure 5 . The dust coats, even when troweled into the surfaces immediately after placing, reduced the depth of wear; but greater reductions were obtained by 


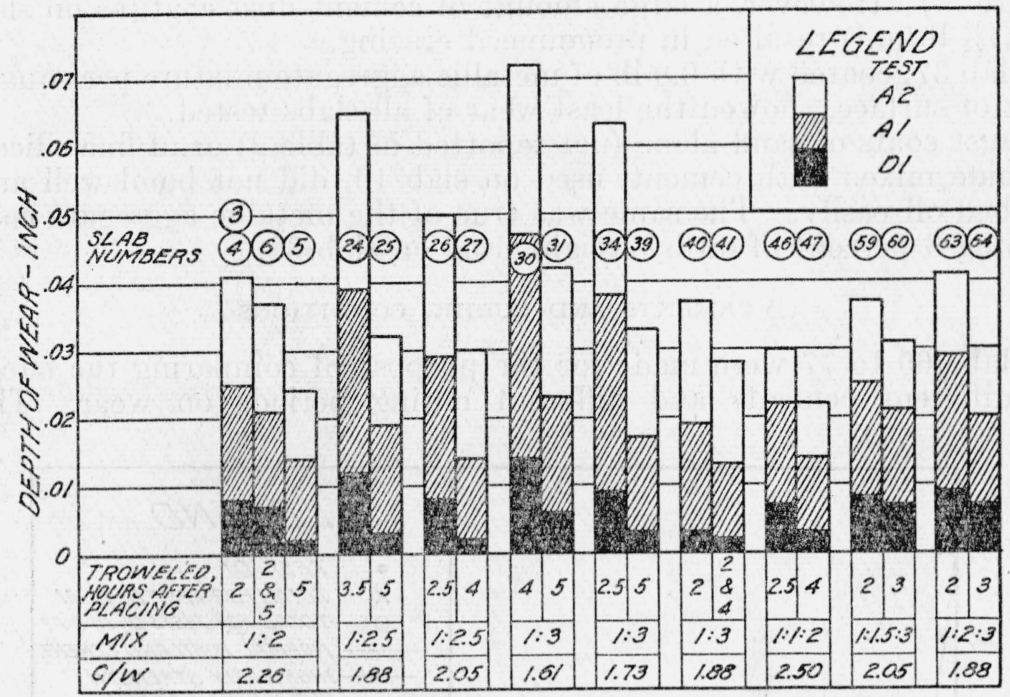

FIGURE 4.-Effect of time interval between placing and troweling.

Test $D 1=5 \mathrm{~min}$, no abrasive.

Test $A 1=1$ st $10 \mathrm{~min}$ with abrasive

Test $A \&=2 \mathrm{~d} 10 \mathrm{~min}$ with abrasive.

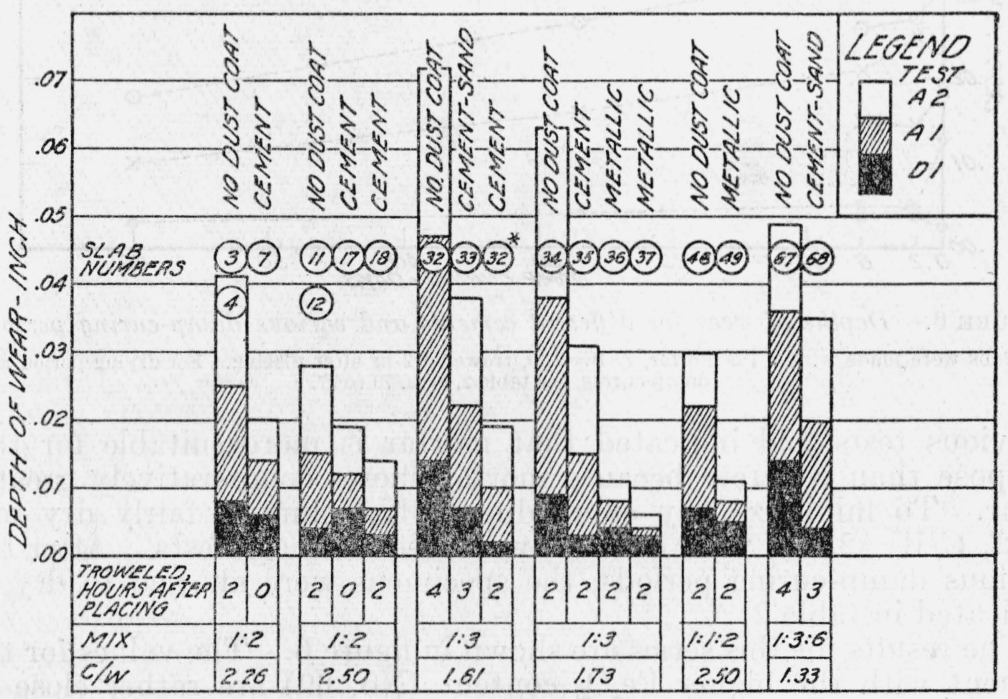

Figure 5.-Effect of dust-coat finishes.

Test $D 1=5 \mathrm{~min}$, no abrasive.

Test $A 1=1$ st 10 min with abrasive.

Test $A Z=2 \mathrm{~d} 10 \mathrm{~min}$ with abrasive.

$\star 11 / 4$ lb cement per sq $\mathrm{ft}$-slab badly crazed. 
delayed troweling. (Compare slabs 11, 17, and 18, in table 2 and figure 5.) However, a large amount of cement dust coat, as on slab $32\left(1 \frac{1}{4} \mathrm{lb} /\right.$ in. $\left.^{2}\right)$ resulted in pronounced crazing.

Slab 37, coated with $0.9 \mathrm{lb}$ of metallic aggregate mixture per square foot of surface, showed the least wear of all slabs tested.

Dust coats of sand alone (not reported in table 2) or of fine silicon carbide mixed with cement, used on slab 19, did not bond well and rubbed off easily. The same was true of the metallic aggregate containing 3 percent of oil by weight used on slab 38 .

\section{(d) CEMENTS AND CURING CONDITIONS}

Slabs 69 to 77 were made for the purpose of comparing the effect of different cements and different curing periods on wear. The

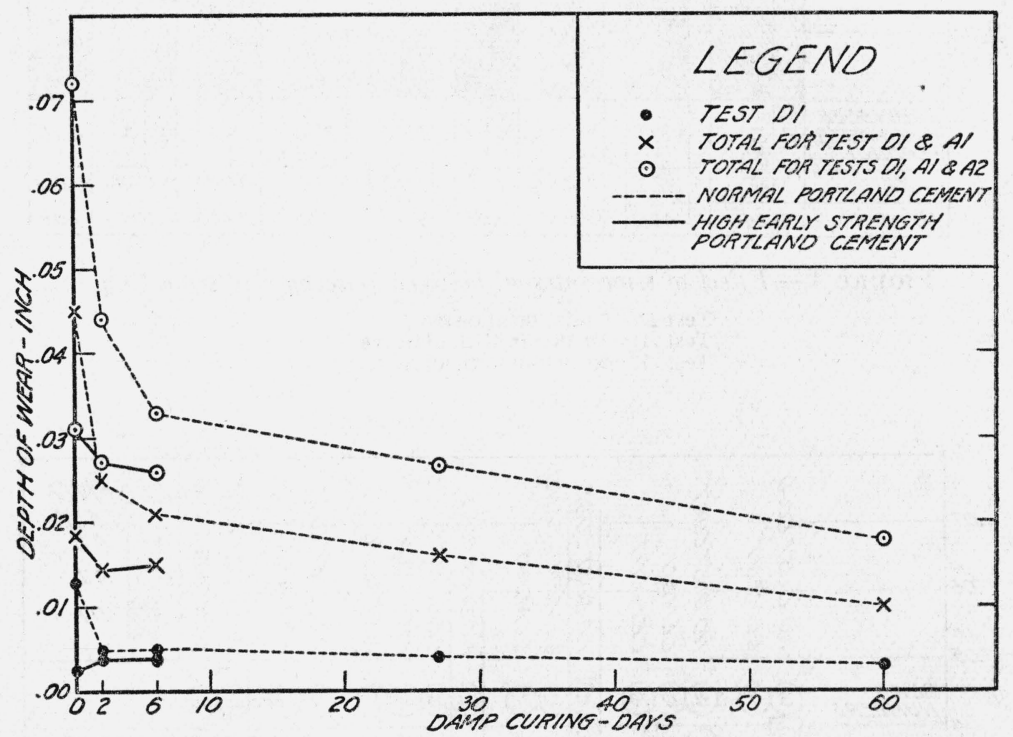

Figure 6.-Depths of wear for different cements and various damp-curing periods. All slabs were made with a 1:2 mortar, $C / W=2.50$, troweled $2 \mathrm{hr}$ after placing. For drying period after damp-curing, see table 2 , Nos. 70 to 77.

previous tests had indicated that mortar is more suitable for this purpose than concrete because mortar shows comparatively greater wear. To minimize any efiect due to troweling, a fairly dry mix $(1: 2, C / W=2.50)$, with sand $B$, was used in these tests. After the various damp-curing periods, the specimens were allowed to dry as indicated in table 2 .

The results for this series are shown in figure 6 . The values for the cement with the higher $\mathrm{Fe}_{2} \mathrm{O}_{3}$ content (No. 69) are rather close to those for the portland cement with less $\mathrm{Fe}_{2} \mathrm{O}_{3}$ and were therefore omitted for clarity of the figure. Without damp-curing, the slabs made with the "normal" cement showed low wear resistance. Dampcuring for only 2 days caused a considerable reduction in depth of wear-about 60 percent in the $D 1$ test, 40 percent in the $A 1$ test, 30 percent in the $A 2$ test, and about 40 percent for the total depth for all three tests. Continued damp-curing caused further reductions; 
after 6 days the reduction in total depth of wear was about 55 percent and, after 60 days, about 75 percent.

The slabs made with the high-early-strength cement showed better wear resistance when not damp-cured than did the slabs with the "normal" cement damp-cured as much as 6 days. Although dampcuring improved the mortar made with the high-early-strength cement, such curing was not as necessary as for the slabs made with the "normal" cement.

Specimens made with the normal cement and not damp-cured (Nos. 13, 73, and 79) showed low wear resistance in the D2 test compared with that in the $D 1$ test, as seen in table 2 for slabs 13 and 79 . For damp-cured specimens the D2 test generally indicated much higher wear resistance than the $D 1$ test, as for slabs 78, 84, 90, and 93.

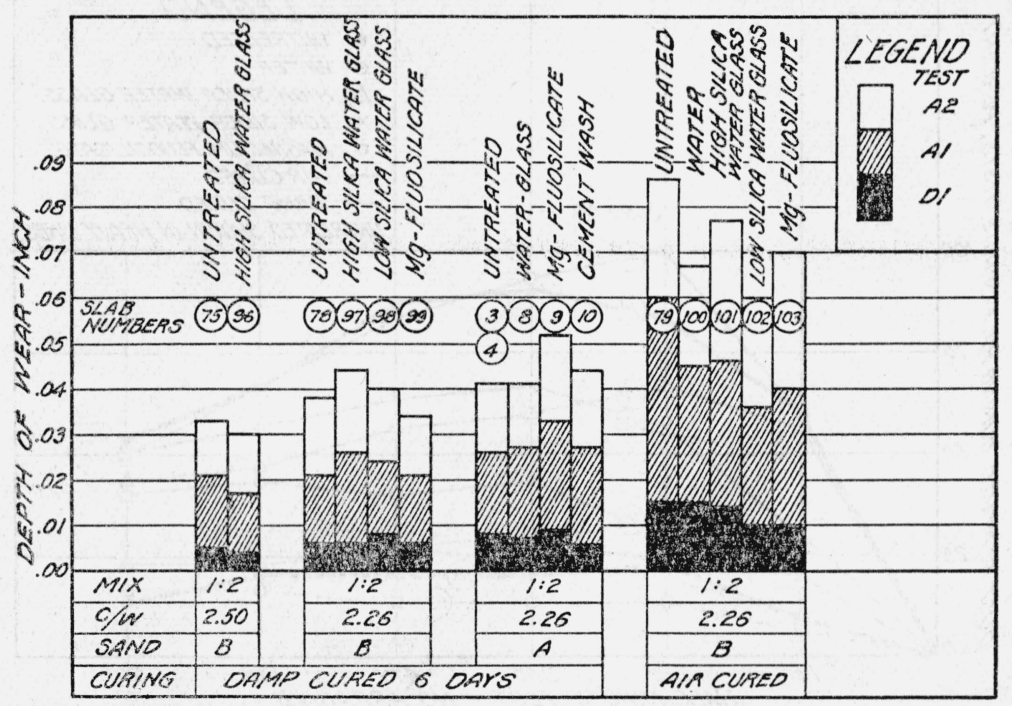

FiguRE 7.-Effect of liquid surface treatments.

All slabs were troweled $2 \mathrm{hr}$ after placing. All surface treatments were applied when slabs were 28 days old. Test $D 1=5 \mathrm{~min}$, no abrasive.

Test $A 1=1$ st 10 min with abrasive.

Test $A 2=2 \mathrm{~d} 10 \mathrm{~min}$ with abrasive.

The wear resistance in the $D 2$ test was also poor for slab 30 , made of wet 1:3 mortar. The $D 1$ test values gave an approximation of the extent of damp-curing under these test conditions when a normal cement was used. Thus, for slab 73, with no damp-curing, $64 \mathrm{~g}$ of dust was abraded in the $D 1$ test, whereas for the corresponding slab, 74 , with 2 days damp-curing, only $27 \mathrm{~g}$ of dust was abraded.

\section{(e) LIQUID SURFACE TREATMENTS}

Eighteen slabs of 1:2 mortar were given liquid surface treatments. All of these slabs were duplicates of other slabs which had been tested without surface treatments. Ten of these were damp-cured for 6 days and eight were stored in the laboratory. All were treated at the age of 28 days.

Figure 7 shows the depths of wear for the treated slabs in the D1, $A 1$, and $A 2$ tests in comparison with the depths of wear of untreated 
slabs. The tests show that the liquid surface treatments did not produce a significant increase in wear resistance of damp-cured slabs but produced an improvement in slabs that had not been dampcured. The application of water instead of the solution reduced the total depth of wear to some extent.

The series of half-minute tests, without abrasive, was made to determine the depths to which the surface treatments were effective. The results, given in figure 8 , show that with three exceptions the greatest weight of dust was removed during the second or third halfminute test. The damp-cured specimens, as a group, showed less wear than those not damp-cured, and the slabs treated with magnesium fluosilicate showed the least wear in each group.

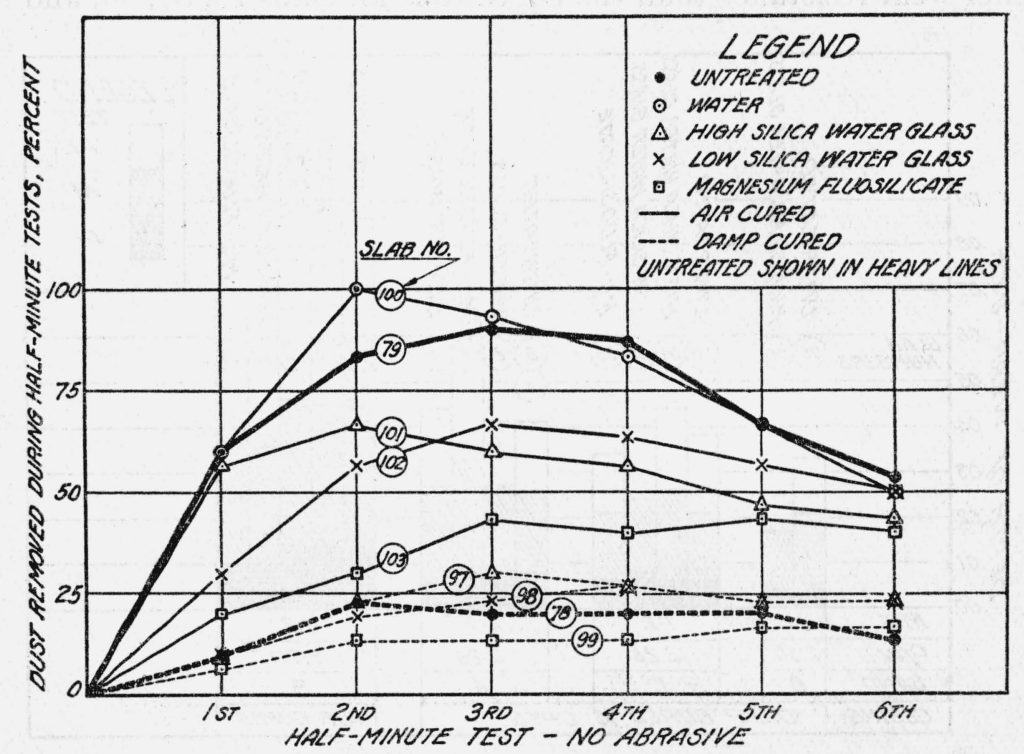

FIGURE 8.-Effect of liquid surface treatments.

All slabs were made with a 1:2 mortar, $C / W=2.26$, troweled $2 \mathrm{hr}$ after placing. The amounts of dust are expressed as percentages of the amount of dust removed from slab 100 during the second half-minute test (3.0 grams).

The tests show that in no case did surface treatments compensate for lack of damp-curing. The total depth of wear for all these halfminute tests was about 0.005 in. for the damp-cured slabs and 0.01 in. for those not damp-cured. At these depths the rates of wear in each group approached a constant value.

\section{PITTING}

The surfaces of the mortar slabs did not remain smooth after a small amount of wear had taken place; nearly all of them were moderately to excessively pitted. The various degrees of pitting are illustrated in figures 9,10 , and 11 , which show portions of the worn surfaces. Because of the manner in which the photographs were taken, the pitting appears more pronounced than it would under ordinary illumination. A surface classed as excessively pitted would probably not be acceptable under any condition. "Moderate pitting" would ordinarily be acceptable except for especially high-grade work. 

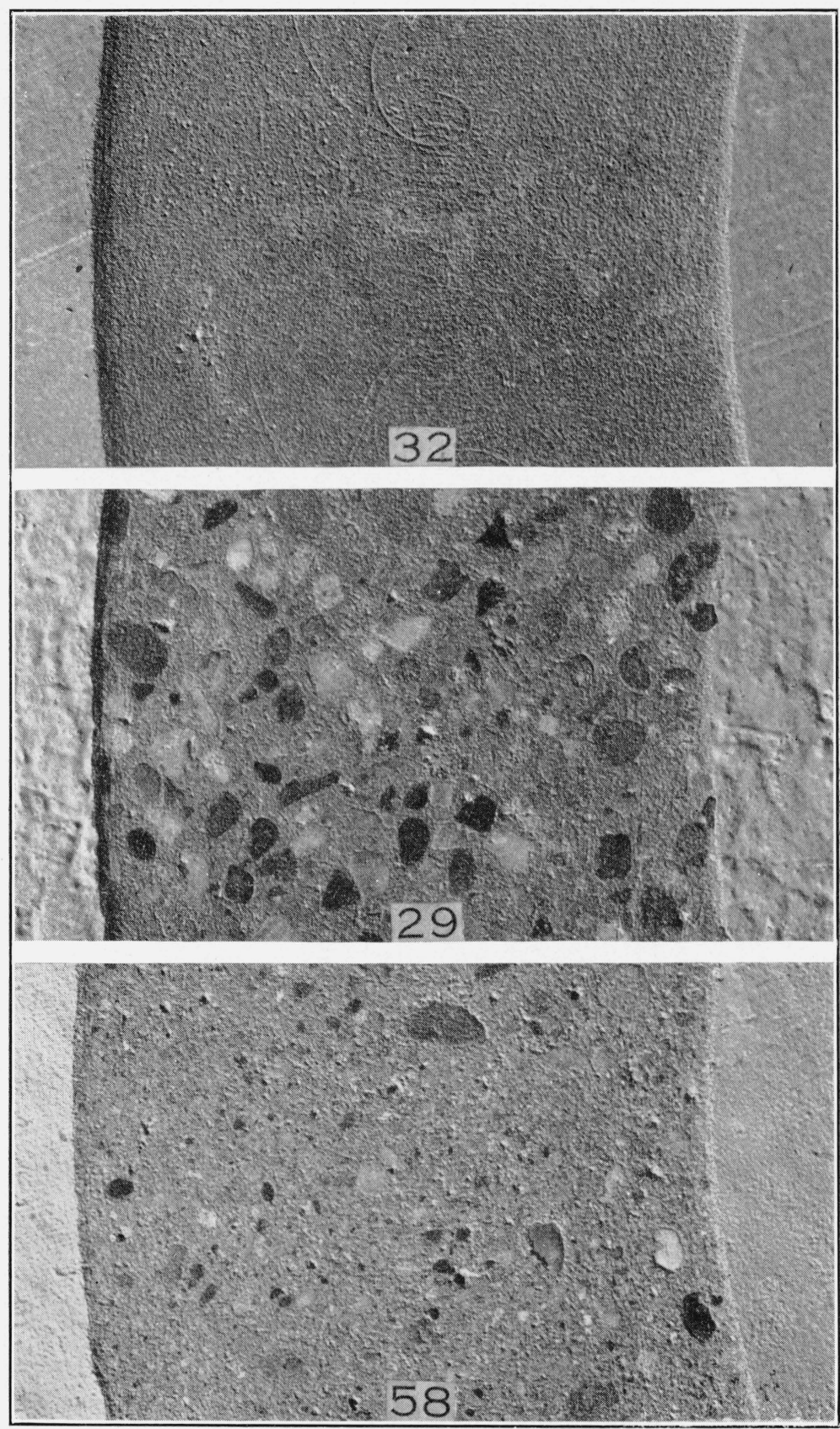

Figure 9.-Appearance of slabs after test, showing degrees of pitting. Slab 32, negligible pitting; slabs 29 and 58 , slight pitting. 


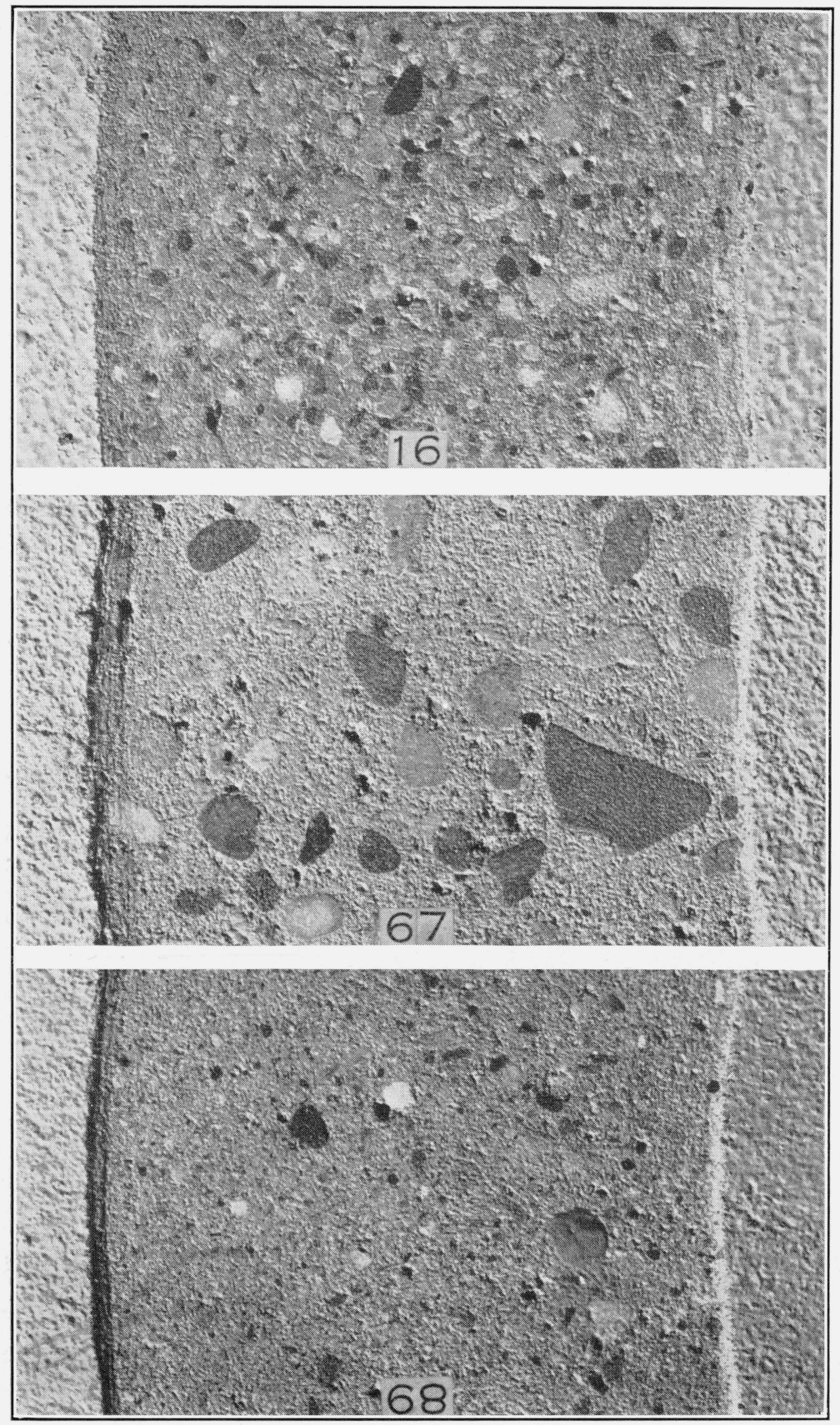

Figure 10.-A ppearance of slabs after test, showing degrees of pitting. Slabs 16,67 , and 68 , slight pitting. 


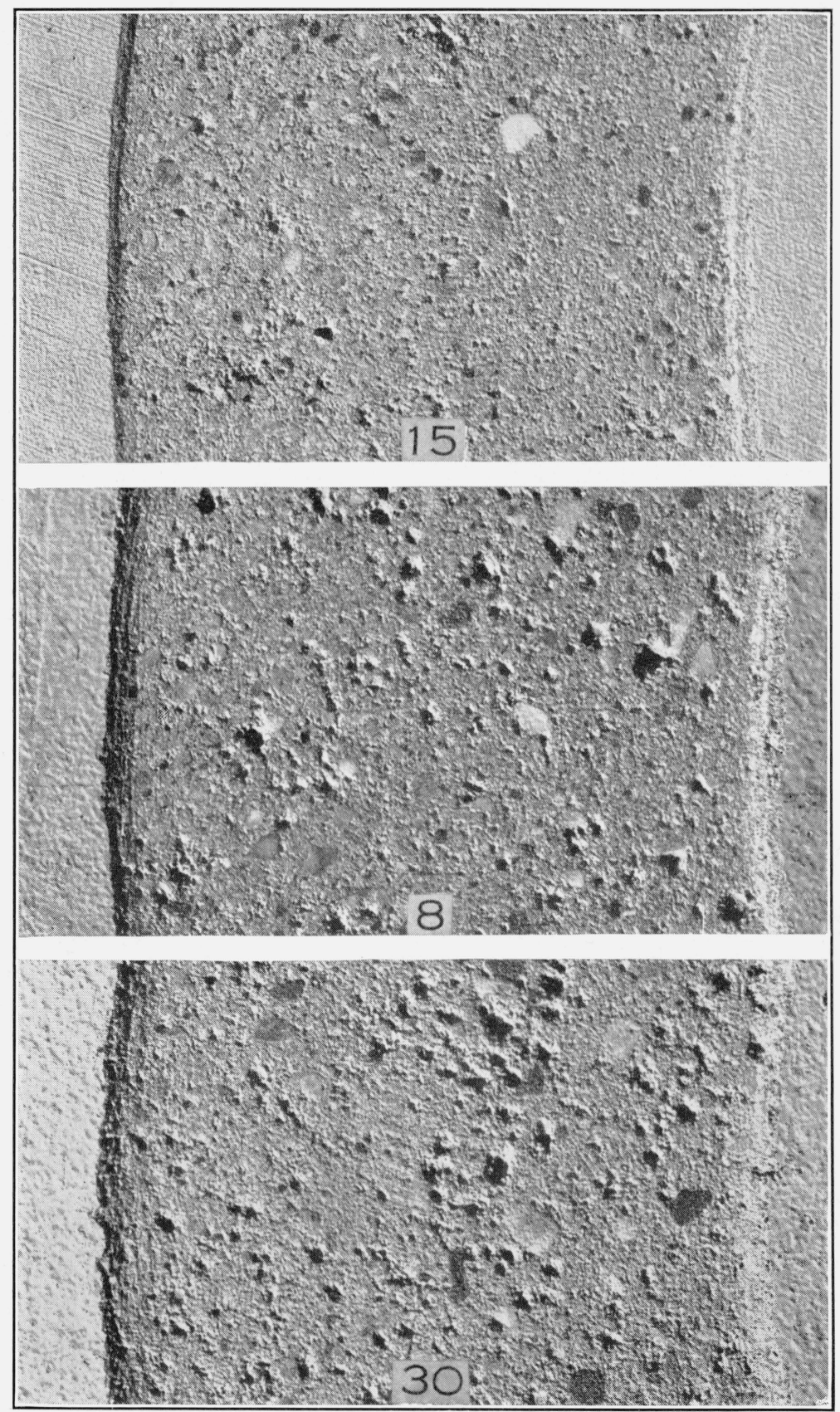

FIGURE 11.-A ppearance of slabs after test, showing degrees of pitting. Slab 15, moderate pitting; slabs 8 and 30 , excessive pitting. 
Pitting may be due to exposure of voids in the mortar or concrete, or to actual loosening of particles of aggregate, as observed by Kessler [8] for some sandstones, in which the grains are comparatively hard but poorly bonded. The slabs in which the pitting after the $A 2$ test was slight were either dry 1:2 mortars or mixes with gravel in which the volume of mixing water was less than 24 percent of that of the concrete. Those slabs which were moderately pitted contained 24 to 28 percent by volume of mixing water. Those excessively pitted included the $1: 3 \frac{1}{2}$ and 1:4 mortars, the mortars with water contents exceeding 28 percent, and mortars made with normal cement but not damp-cured-Nos. 13, 73, and 79. Generally the mixes which were badly pitted also showed comparatively poor wear resistance.

Dust coats reduced the degree of pitting, especially when applied to mortars; compare slabs 3 and $7 ; 11$ and $17 ; 30,32$, and 33 (table 2). The only slabs in which the pitting was negligible were two to which dust coats had been applied (slab 32 with a dust coat of cement, and slab 37 with a metallic aggregate-cement dust coat). However, when the metallic aggregate contained oil (3 percent by weight), a slab (No. 38) to which it was applied showed excessive pitting.

\section{SUMMARY AND CONCLUSIONS}

A portable testing apparatus for producing rapid abrasive wear of concrete floor surfaces and a device for measuring depths of wear were developed. The apparatus is placed directly on the surface to be tested and may be used with or without an added abrasive.

The test methods were applied in a study of concrete slabs 1 to 2 in. thick, by $23 \frac{1}{2}$ by $27 \frac{1}{2}$ in., and made of mixes varying from a $1: 2$ mortar to a $1: 3: 6$ concrete. The $C / W$ ratios, aggregate types and gradings, time intervals between placing and troweling, and curing conditions were varied. Surface treatments including liquid "hardeners" and metallic aggregates were also studied.

The dependability of the apparatus and method used for measuring the wear was determined by computing the standard deviations of the wear values. The computations showed that variations between the wear values of slabs made in duplicate were of the same magnitude as would be expected from the variations in eight individual measurements made on a single slab, indicating satisfactory reproducibility in slabs. The reproducibility of the test results as determined from the standard deviations indicated that the difference in wear values obtained for different slabs was significant and that the apparatus could distinguish the effect of variations in mixes, treatments, curing, and so forth.

A 5-minute test under the wearing action of steel disks, without an abrasive, shows large variations in wear resistance for various mixes, $C / W$ ratios, time intervals between placing and troweling, and treatments such as dust coats or liquid surface treatments. The weight of dust removed in the test varied from 6 to $81 \mathrm{~g}$, and the depth of wear from 0.002 to 0.018 in. The slabs showing the greatest wear in this test were those upon which excess water appeared on the surface during or after finishing.

After the 5-minute test, the resistance to wear of the subsurface was determined by two 10 -minute tests with an added abrasive. The depths of wear during the first of these 10-minute tests varied from 0.001 to $0.045 \mathrm{in}$. 
Nearly all of the slabs were more or less pitted after the smooth surface had been worn off. The mortars generally showed more pitting than the concretes.

The following conclusions may be drawn from the test results:

1. For the same mix proportions, the wear resistance of concretes and mortars is greater for higher $C / W$ ratios. For equal $C / W$ ratios, wear resistance increases with increasing proportion of aggregates up to a certain point, depending on the aggregates used, then decreases. Some mixes which had poor wear resistance at the surface were relatively more resistant after the surface "skin" was removed.

2. The shape of the aggregate particles (rounded or angular) affects the amount of water required for ease of placing and finishing, and thus may affect wear resistance of the concrete as much as the abrasion resistance of the particles themselves.

3 . In this investigation, delaying the troweling for 3 or more hours after placing the concrete tended to increase wear resistance, especially for mixes containing no coarse aggregate.

4. The wear resistance of many mixes is greatly improved by troweling into the surfaces dust coats of cement, of cement and sand, or of cement and metallic aggregate. For example, the slab to which was applied a heavy dust coat consisting of a mixture of two parts of metallic aggregate to one of cement, by weight, had the highest wear resistance of all slabs tested. A dust coat also facilitates finishing the surface of concrete made of a lean mix, such as 1:3:6.

5. Concrete made with a "normal" portland cement and aged in the air without damp-curing may show comparatively poor wear resistance; but damp-curing may increase the wear resistance appreciably. Where a high-early-strength cement is used, the dampcuring is not as necessary.

6. Liquid surface treatments, such as solutions of magnesium fluosilicate or of water glass, are effective in improving the wear resistance of concrete that has not been damp-cured.

7. The use of coarse aggregates, such as gravel, permits reduction in the water content of the mixes and helps to reduce pitting. The use of dust coats containing cement also helps to reduce pitting, especially when applied to lean mixes.

\section{REFERENCES}

[1] Report of Service Tests on Concrete Floor Treatments, Letter Circular NBS LC139 (Oct. 28, 1920).

[2] D. A. Abrams, Wear tests of concrete, Proc. Am. Soc. Testing Materials 21, pt. 2, 13 (1921).

[3] C. E. Covell, Heavy duty concrete floors, Proc. Am. Concrete Inst. 27, 454 (1928).

[4] Jackson and Pauls, Accelerated wear tests of concrete pavements, Proc. Am. Soc. Testing Materials 24, pt. 2, 864.

[5] Ahlers, Linden, and Bird, Wear tests on floor finishes at warehouse of $R . H$. Macy Co., Long Island, N. Y., Proc. Am. Concrete Inst. 24, 778 (1929).

[6] J. G. Ahlers, Good practice in concrete floor finish, Proc. Am. Concrete Inst. 26, $520(1930)$

[7] W. E. Hart, Effect of materials and manipulation upon quality of finish, Proc. Am. Concrete Inst. 26, 532 (1930).

[8] D. W. Kessler, Wear resistance of natural stone flooring, BS J. Research 11, 635 (1933) RP612.

Washington, March 1, 1939. 